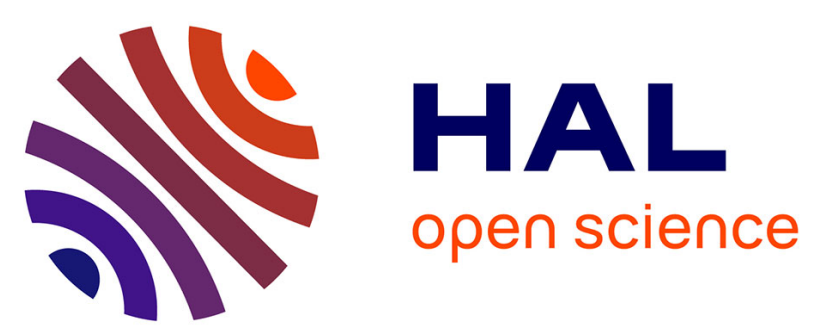

\title{
Bernstein-Gel'fand-Gel'fand reciprocity and indecomposable projective modules for classical algebraic supergroups
}

Caroline Gruson, Vera Serganova

\section{To cite this version:}

Caroline Gruson, Vera Serganova. Bernstein-Gel'fand-Gel'fand reciprocity and indecomposable projective modules for classical algebraic supergroups. Moscow Mathematical Journal, 2013, 13 (2), pp.281-313. 10.1007/978-1-4471-4863-0_7 . hal-01093230

\section{HAL Id: hal-01093230 \\ https://hal.science/hal-01093230}

Submitted on 10 Dec 2014

HAL is a multi-disciplinary open access archive for the deposit and dissemination of scientific research documents, whether they are published or not. The documents may come from teaching and research institutions in France or abroad, or from public or private research centers.
L'archive ouverte pluridisciplinaire HAL, est destinée au dépôt et à la diffusion de documents scientifiques de niveau recherche, publiés ou non, émanant des établissements d'enseignement et de recherche français ou étrangers, des laboratoires publics ou privés. 


\title{
Bernstein-Gel'fand-Gel'fand reciprocity and indecomposable projective modules for classical algebraic supergroups
}

\author{
Caroline Gruson ${ }^{1}$ and Vera Serganova ${ }^{2}$
}

\section{To the Memory of I. M. Gelfand}

\begin{abstract}
We prove a BGG type reciprocity law for the category of finite dimensional modules over algebraic supergroups satisfying certain conditions. The equivalent of a standard module in this case is a virtual module called Euler characteristic due to its geometric interpretation. In the orthosymplectic case, we also describe indecomposable projective modules in terms of those Euler characteristics.

Key words: Finite dimensional representations of algebraic supergroups, Flag variety, BGG reciprocity law.
\end{abstract}

\section{INTRODUCTION}

In many representation theories, there exist reciprocity laws. Roughly speaking, if the category in question has enough projective modules, one defines in a natural way a family of so-called standard modules such that every projective indecomposable module has a filtration with standard quotients. The reciprocity law states that the multiplicity of a standard module in the projective cover of a simple module equals the multiplicity of this simple module in the standard module. Those standard modules are usually easy to describe, in particular, their characters are given by simple formulae.

For instance, Brauer discovered such a law in the case of finite groups representations in positive characteristic, [5]. Another example is a result of Humphreys, [13], for representations of semi-simple Lie algebras in positive characteristic. In 1976 ([2]) Bernstein, Gel'fand and Gel'fand introduced the category $\mathcal{O}$ of highest weight modules for a semisimple Lie algebra in characteristic 0 , and proved a reciprocity law in this category. Irving, [14], and Cline, Parshall and Scott, [11], introduced a general notion of highest weight category and proved a generalized BGG reciprocity. Using this general approach, it is easy to prove similar results for the category $\mathcal{O}$ of highest weight modules for classical simple Lie superalgebras. For the category of finite-dimensional representations of classical Lie superalgebras of type I, Zou proved BGG reciprocity in [30]. For superalgebras of type II the question remained open, in particular since it was unclear how to define a standard object.

The first part of this paper (Section 2) is devoted to the generalized BGG reciprocity for algebraic supergroups $G$ with reductive even part and symmetric root decomposition. This class includes all simple supergroups such that the corresponding Lie superalgebras admit an invariant even symmetric form, in particular, both type I and type II classical Lie superalgebras. In those cases, the irreducible representations are parametrized

\footnotetext{
${ }^{1}$ Université de Lorraine, U.M.R. 7502 du CNRS, Institut Elie Cartan, 54506 Vandoeuvre-les-Nancy Cedex, France. E-mail: Caroline.Gruson@iecn.u-nancy.fr

${ }^{2}$ Department of Mathematics, University of California, Berkeley, CA, 94720-3840 USA. E-mail: serganov@math.berkeley.edu
} 
by a highest weight, and if $\lambda$ is a highest weight, we denote by $L_{\lambda}$ the corresponding irreducible representation. Every $L_{\lambda}$ has an indecomposable projective cover $P_{\lambda}$ in the category of finite-dimensional representations of $G,[26]$. However, in this situation there is no direct analogue of the so-called standard modules. Hence we introduce a family of virtual modules $\mathcal{E}(\mu)$, living in the Grothendieck group of the category: we call those modules Euler characteristics because they come from the cohomology of line bundles on flag supervarieties. It turns out that in the Grothendieck ring, the class $\left[P_{\lambda}\right]$ of $P_{\lambda}$ are linear combinations of $\mathcal{E}(\mu)$-s and we denote the coefficient of $\mathcal{E}(\mu)$ in $\left[P_{\lambda}\right]$ by $a(\lambda, \mu)$. In general the coefficients $a(\lambda, \mu)$ may be negative. The reciprocity law (Theorem 1 ) states that $a(\lambda, \mu)$ is exactly the multiplicity of $L_{\lambda}$ in $\mathcal{E}(\mu)$. The key argument in the proof is a $\mathbb{Z} / 2 \mathbb{Z}$-graded analogue of the Bott reciprocity result, [3], see Proposition 1.

All the constructions above depend on the choice of a Borel subgroup in $G$ : in the super case, this choice is not unique up to conjugation, and the result is true for every possible choice. In particular, in the case of $G L(m, n)$ our result generalizes Zou's result. In this case the modules $\mathcal{E}(\mu)$ are not virtual - they coincide with the so-called Kac modules (see the example at the end of Section 2).

It is worth mentioning that in general the weights $\lambda$ (labeling $L_{\lambda}$ and $P_{\lambda}$ ) and $\mu$ (labeling $\mathcal{E}(\mu)$ ) do not belong to the same set. For instance, in the orthosymplectic case (Section 4) the $\mu$-s must have tailless weight diagrams. Finally, let us emphasize on the fact that this category has infinite cohomological dimension and the subgroup generated by $\left[P_{\lambda}\right]-\mathrm{s}$ is a proper subgroup in the whole Grothendieck group. Probably the simplest example of such situation is the category of finite-dimensional representations of the algebra $\mathbb{C}[z] /\left(z^{2}\right)$ with a unique simple module $L$ and a unique indecomposable projective module $P$ related by $[P]=2[L]$ in the Grothendieck group.

The rest of the paper deals with the computation of the coefficients $a(\lambda, \mu)$ for the orthosymplectic supergroup $\operatorname{SOSP}(m, 2 n)$. The first computation of those coefficients in the $G L(m, n)$ case was made in [24]. In [4], J. Brundan used another method, relating this representation theory with the one of $\mathfrak{g l}_{\infty}$. He interpreted the translation functors for $\mathfrak{g l}(m, n)$ as linear operators of $\mathfrak{g l}_{\infty}$ acting on $\Lambda^{n}(V) \otimes \Lambda^{m}\left(V^{*}\right)$, where $V$ is the standard representation of $\mathfrak{g l}_{\infty}$. Later on, in $[6,7,8]$ Brundan and Stroppel introduced weight diagrams, which give a clear picture of the translation functors action. Thus the category of finite dimensional $G L(m, n)$-modules is very well understood now, including the projective modules.

We adopt Brundan's categorification approach. Here we have to separate in two cases depending on the parity of $m$. If $m$ is odd, we identify the lattice in the Grothendieck group generated by $\mathcal{E}(\mu)$-s with a natural lattice in the tensor representation $\Lambda^{m}\left(V^{*}\right) \otimes \Lambda^{n}(V)$ of the infinite-dimensional Lie algebra $\mathfrak{g l}_{\infty / 2}$ with Dynkin diagram

$$
\circ-\circ-\circ-\ldots
$$

As in the case of $G L(m, n)$ certain translation functors correspond to the Chevalley generators of $\mathfrak{g l}_{\infty / 2}$. However, there is another translation functor, which we call the switch functor which does not have such interpretation. We compute the coefficients $a(\lambda, \mu)$ in Section 8 (see Theorem 2, Theorem 3 and Theorem 4) via a comparison between the action of the translation functors on $P_{\lambda \text {-s }}$ and $\mathcal{E}(\mu)$-s. We start with a typical $\lambda$ (in this case $P_{\lambda}, \mathcal{E}(\lambda)$ and $L_{\lambda}$ coincide) and then obtain an arbitrary $P_{\lambda}$ by application of translation functors. If $m$ is even, the corresponding infinite-dimensional Lie algebra is $\mathfrak{g l}_{\infty / 2} \oplus \mathfrak{g l}_{\infty / 2}$ (see Section 7). 
Note that in [4] Brundan identifies indecomposable projectives with certain topological basis in a natural completion of the tensor representation of $\Lambda^{m}\left(V^{*}\right) \otimes \Lambda^{n}(V)$. In our case one can realize projectives inside $\Lambda^{m}\left(V^{*}\right) \otimes \Lambda^{n}(V) \otimes_{\mathbb{Z}} \mathbb{Q}$ without taking completion, see Proposition 3 (Section 8). In particular, one can express any $\mathcal{E}(\mu)$ as a finite rational combination of $\left[P_{\lambda}\right]$-s. This is an essential difference with the case of $G L(m, n)$. One only has to use the completion for realization of simple modules as we explain at the end of paper. At the moment we do not have a nice characterization of the lattice in the completion generated by the classes of simple modules.

The main result of the paper for orthosymplectic supergroups is a simple combinatorial algorithm calculating $a(\lambda, \mu)$. This algorithm implies, in particular, that $a(\lambda, \mu)=0, \pm 1$. It also provides the algorithm for calculating characters of all indecomposable finitedimensional projective modules and multiplicities of all finite-dimensional simple modules in all indecomposable projective modules. That however does not imply automatically an expression of irreducible characters in the same terms. In the case of $G L(m, n)$ this difficulty can be resolved by allowing infinite linear combinations of Euler characteristics $\mathcal{E}(\mu)$-s, i.e. by completing the Grothendieck ring. For general approach in this situation see [1]. It is possible to do in our case, but we do not solve this problem in the present paper. We only give an illustration how it can be done in the simplest case (see the example at the end of the paper).

The problem of calculating irreducible characters was solved in [12] by a slightly different method, namely, by calculating Euler characteristics of vector bundles over an adequate variety (a generalized grassmannian) related to the highest weight and using an induction on the rank of the supergroup. It seems that this difference between general linear and orthosymplectic cases is related to the fact that in the latter case the set of dominant weights has a minimal element with respect to the standard order.

There remain several open questions such as an interpretation of indecomposable projectives and simple modules in terms of canonical bases and the construction of the analogue of Khovanov's diagram algebra, see [6],[7], [8] and [9]. It would be also quite interesting to understand how formulae for characters of the projective modules obtained in this paper are related to the results of [10]. In Section 4 we explain how to transfer combinatorial data of [10] into the language of weight diagrams used in the present paper.

We thank Jonathan Brundan, Catharina Stroppel and Elizaveta Vishnyakova for fruitful discussions. This work was partially supported by NSF grant n. 0901554.

\section{Notations And CONTEXT}

We work over algebraically closed field of characteristic zero. For the general theory of Lie superalgbras and their representations, see [20]. Let $G$ be a connected algebraic supergroup with reductive even part $G_{0}$ and $\mathfrak{g}$ denote its Lie superalgebra. Then $\mathfrak{g}_{0}$ is a a reductive Lie algebra and $\mathfrak{g}$ is a semisimple $\mathfrak{g}_{0}$-module, see [26]. We denote by $\mathfrak{h}_{0}$ a Cartan subalgebra of $\mathfrak{g}_{0}$ and by $\mathfrak{h}$ a Cartan subalgebra of $\mathfrak{g}$, let $H_{0}$ and $H$ be the corresponding algebraic subgroups of $G$. Denote by $W$ the Weyl group $W\left(\mathfrak{g}_{0}, \mathfrak{h}_{0}\right)$.

In order to prove the BGG reciprocity we need the following assumptions on $\mathfrak{g}$

- $\mathfrak{h}=\mathfrak{h}_{0}$, and therefore $H=H_{0}$;

- $\mathfrak{g}_{1} \simeq \mathfrak{g}_{1}^{*}$ as a $\mathfrak{g}_{0}$-module.

The following simple Lie superalgebras satisfy these assumptions: $(\mathfrak{p}) \mathfrak{s l}(m, n), \mathfrak{o s p}(m, 2 n)$, $D(2,1 ; a), G_{3}$ and $F_{4}$. Other examples can be found in [26]. 
Recall that $H=H_{0}$ is an algebraic torus. Let $\Lambda$ denote the free abelian group of characters of $H$. One has a root decomposition

$$
\mathfrak{g}=\mathfrak{h} \oplus \bigoplus_{\alpha \in \Delta} \mathfrak{g}_{\alpha}
$$

where

$$
\mathfrak{g}_{\alpha}=\left\{x \in \mathfrak{g} \mid \operatorname{Ad}_{h} x=\alpha(h) x, \forall h \in H\right\} .
$$

The finite subset $\Delta \subset \Lambda$ is called the set of roots of $\mathfrak{g}$. Our assumptions imply that $\operatorname{dim} \mathfrak{g}_{\alpha}=(1,0)$ or $\left(0, m_{\alpha}\right)$ for any root $\alpha \in \Delta$. In the former case we say that $\alpha$ is even and in the latter that $\alpha$ is odd. So we have a decomposition $\Delta=\Delta_{0} \cup \Delta_{1}$ defined by the parity of roots. Furthermore, our assumptions imply that $\Delta=-\Delta$. It is not difficult to show that one can define a parity function $p: \Lambda \rightarrow \mathbb{Z}_{2}$ satisfying the condition $p(\lambda+\alpha)=p(\lambda)+p(\alpha)$ for all $\lambda \in \Lambda$ and $\alpha \in \Delta$. In general the choice of $p$ is not unique.

As in the case of reductive Lie algebras, we define a decomposition $\Delta=\Delta^{+} \cup \Delta^{-}$of roots and the corresponding triangular decomposition

$$
\mathfrak{g}=\mathfrak{n}^{-} \oplus \mathfrak{h} \oplus \mathfrak{n}
$$

where

$$
\mathfrak{n}^{-}=\bigoplus_{\alpha \in \Delta^{-}} \mathfrak{g}_{\alpha}, \mathfrak{n}=\bigoplus_{\alpha \in \Delta^{+}} \mathfrak{g}_{\alpha}
$$

The subalgebra $\mathfrak{b}=\mathfrak{h} \oplus \mathfrak{n}$ is called a Borel subalgebra of $\mathfrak{g}$ and the corresponding algebraic subgroup $B$ is called a Borel subgroup of $G$. Recall that the Borel subgroups in $G$ are not always mutually conjugate. It is easy to see that $B$ is the semi-direct product of the algebraic torus $H$ and the unipotent supergroup $N$. We set

$$
\rho_{0}=\frac{1}{2} \sum_{\alpha \in \Delta_{0}^{+}} \alpha, \rho_{1}=\sum_{\alpha \in \Delta_{1}^{+}} m_{\alpha} \alpha, \quad \rho=\rho_{0}-\rho_{1} .
$$

By $\mathcal{C}$ we denote the category of finite-dimensional $G$-modules, which is isomorphic to the category of $\mathfrak{g}$-modules, semisimple over $\mathfrak{h}$, with weights in $\Lambda$, see [26]. It was shown in [17] that any simple module in $\mathcal{C}$ is a quotient of a Verma module with highest weight $\lambda \in \Lambda$ by its maximal submodule. A weight $\lambda$ is called dominant if this quotient is finitedimensional. Thus, every dominant weight defines two simple modules, one is obtained from another by application of the functor $\Pi$ of change of parity. In order to avoid parity chasing, we introduce a parity function $p: \Lambda \rightarrow \mathbb{Z}_{2}$ and define the category $\mathcal{F}$ as the full subcategory of $\mathcal{C}$ consisting of modules such that the parity of any weight space coincides with the parity of the corresponding weight. It is not hard to see that $\mathcal{C}=\mathcal{F} \oplus \Pi(\mathcal{F})$.

Define the standard order on $\Lambda$ by setting: $\lambda \leq \mu$ iff $\mu-\lambda=\sum_{\alpha \in \Delta^{+}} n_{\alpha} \alpha$ where all $n_{\alpha}$ are non-negative integers.

For any dominant weight $\lambda$, we will denote by $L_{\lambda}$ the simple $\mathfrak{g}$-module in the category $\mathcal{F}$ with highest weight $\lambda$.

It is well-known (see, for example, [26] Lemma 9.1), that in the category $\mathcal{F}$ every simple module $L_{\lambda}$ has an indecomposable projective cover which we denote by $P_{\lambda}$.

Denote by $\Lambda^{+}$the set of all weights $\lambda$ such that $\langle\lambda, \breve{\beta}\rangle$ is a positive integer for any simple $\operatorname{root} \beta$ of $\Delta_{0}^{+}$, where $\check{\beta} \in\left[\mathfrak{g}_{\beta}, \mathfrak{g}_{-\beta}\right]$ such that $\langle\beta, \breve{\beta}\rangle=2$.

Let $\mathcal{R}=\mathbb{Z}\left[e^{\mu}\right]$ for all $\mu \in \Lambda$. For $M \in \mathcal{F}$ let $m_{\mu}$ denote the multiplicity of the weight $\mu$ in $M$. Define the character

$$
C h(M)=\sum_{\mu \in \Lambda} m_{\mu} e^{\mu} \in \mathcal{R}
$$


Denote by $\mathcal{K}(\mathcal{F})$ the Grothendieck ring of the category $\mathcal{F}$ (multiplication comes from tensor product). We will denote by $[M]$ the class of a module $M$ in $\mathcal{K}(\mathcal{F})$ and by $[M: L]$ the multiplicity of an irreducible module $L$ in the module $M$. Clearly, $\left[L_{\lambda}\right]$ for all dominant $\lambda$ form a basis of $\mathcal{K}(\mathcal{F})$. It is also clear that $C h: \mathcal{K}(\mathcal{F}) \rightarrow \mathcal{R}$ is a homomorphism of rings. Note that

$$
C h\left(L_{\lambda}\right)=e^{\lambda}+\sum_{\mu<\lambda} m_{\mu} e^{\mu}
$$

thus the $C h\left(L_{\lambda}\right)$ are linearly independent. Due to our convention about parity, for any two modules $M$ and $N$ in $\mathcal{F}, C h(M)=C h(N)$ if and only if $[M]=[N]$ in $\mathcal{K}(\mathcal{F})$. Hence $C h$ is injective. For classical Lie superalgebras the image of $C h$ is described in [29].

Let $\mathfrak{g}$ be a finite-dimensional Kac-Moody superalgebra such that the quotient of $[\mathfrak{g}, \mathfrak{g}]$ by the center is simple. In other words, $\mathfrak{g}=\mathfrak{s l}(m, n)$, where $1 \leq m<n, \mathfrak{g l}(n, n)$ with $n \geq 2, \mathfrak{o s p}(m, 2 n), D(2,1 ; \alpha), G_{3}$ or $F_{4}$ (see [16]). Let $G$ denote a connected algebraic supergroup with Lie algebra $\mathfrak{g}$. For existence of such supergroup see, for instance, [26]. Then $G$ satisfies our assumptions.

Recall that $\mathfrak{g}$ is equipped with a non degenerate invariant bilinear form and the restriction of this bilinear form to $\mathfrak{h}$ is also non degenerate. Thus, we have a non-degenerate form on $\Lambda$.

Following [17], we call a weight $\lambda$ typical if $(\lambda+\rho, \alpha) \neq 0$ for any isotropic root $\alpha$. (Recall that an isotropic root is automatically odd). It follows from [17] that $P_{\lambda}=L_{\lambda}$ if and only if $\lambda$ is typical.

As follows from [17] a typical $\lambda$ is dominant iff $\lambda+\rho \in \Lambda^{+}$. For a general $\lambda$, the latter statement is true only for $\mathfrak{g l}(m, n)$ or $\mathfrak{o s p}(2,2 n)$ and a special choice of a Borel subgroup.

Let $\mathcal{U}(\mathfrak{g})$ be the universal enveloping algebra of $\mathfrak{g}$ and $\mathcal{Z}(\mathfrak{g})$ be its center. For every weight $\lambda$, we write $\chi_{\lambda}$ for the corresponding central character. A central character $\chi$ is dominant if there exists a dominant $\lambda$ such that $\chi=\chi_{\lambda}$.

The category $\mathcal{F}$ splits into direct sum of blocks $\oplus \mathcal{F}_{\chi}$ consisting of modules admitting the generalized central character $\chi$. For any $M \in \mathcal{F}$ we denote by $M_{\chi}$ the projection of $M$ to the block $\mathcal{F}_{\chi}$.

\section{Geometric induction and BGG Reciprocity}

In this section we assume that $G$ satisfies the assumptions of Section 1 .

Let $B$ be a Borel subgroup of $G$ with Lie algebra $\mathfrak{b}$, and let $V$ be a $B$-module. In [12] we defined a derived functor $\Gamma_{i}(G / B, \cdot)$ from the category of $B$-modules to the category of $G$-modules. For any $B$-module $V$ we define

$$
\Gamma_{i}(G / B, V):=H^{i}\left(G / B, V^{*}\right)^{*},
$$

where $H^{i}\left(G / B, V^{*}\right)$ denotes the $i$-th cohomology group of the induced vector bundle $G \times{ }_{B} V^{*}$ on the flag supervariety $G / B$ (see [18],[19] and [22] for definitions). Recall that $H^{i}\left(G / B, V^{*}\right)$ is a $G$-module, see [18], [19], and it is not difficult to see that $\Gamma_{i}(G / B, V):=$ $H^{i}\left(G / B, V^{*}\right)^{*}$ is an object of $\mathcal{F}$ if $V$ satisfies the parity condition about weights.

It is also possible to define $H^{i}\left(G / B, V^{*}\right)$ following [15]. Using this approach one can avoid the rather technical question of existence of $G / B$. We will follow this approach in the paper. We define $H^{0}(G / B, \cdot)$ as a functor from the category of $B$-modules to the category of $G$-modules using the Zuckerman functor, see, for instance, [27]. Let $\Gamma_{\mathfrak{g}_{0}}(M)$ denote the set of $\mathfrak{g}_{0}$-finite vectors in a $\mathfrak{g}$-module $M$. For any $B$-module $V$ we set

$$
H^{0}(G / B, V)=\Gamma_{\mathfrak{g}_{0}}\left(\operatorname{Hom}_{U(\mathfrak{b})}(U(\mathfrak{g}), V)\right) .
$$


It is not hard to see that $H^{0}(G / B, V)$ has a unique $G$-module structure compatible with the action of $\mathfrak{g}$. It is straightforward that $H^{0}(G / B, \cdot)$ is right adjoint to the restriction functor. Furthermore, $H^{0}(G / B, \cdot)$ is left exact, and we define $H^{i}(G / B, \cdot)$ as the right derived functor of $H^{0}(G / B, \cdot)$.

Denote by $C_{\lambda}$ the one-dimensional $B$-module with weight $\lambda \in \Lambda$ and by $\mathcal{E}(\lambda)$ the class of the Euler characteristic

$$
\mathcal{E}(\lambda):=\sum_{\mu \in \Lambda} \sum_{i=0}^{\operatorname{dim}(G / B)_{0}}(-1)^{i}\left[\Gamma_{i}\left(G / B, C_{\lambda}\right): L_{\mu}\right]\left[L_{\mu}\right] .
$$

One can easily generalize Proposition 1 in [12] or Theorem 12 in [27] and obtain the following character formula for $\mathcal{E}(\lambda)$

\section{Lemma 1. -}

$$
C h \mathcal{E}(\lambda)=D \sum_{w \in W} \varepsilon(w) e^{w(\lambda+\rho)}
$$

where

$$
D_{0}=\prod_{\alpha \in \Delta_{0}^{+}}\left(e^{\alpha / 2}-e^{-\alpha / 2}\right), D_{1}=\prod_{\alpha \in \Delta_{1}^{+}}\left(e^{\alpha / 2}+e^{-\alpha / 2}\right)^{m_{\alpha}}, D=\frac{D_{1}}{D_{0}} .
$$

Proof. - Note that in the case $G=G_{0},(2)$ is a direct consequence of the Borel-Weil-Bott theorem. Moreover, if $V$ is a $B_{0}$-module and

$$
\mathcal{E}_{0}(V):=\sum_{i=0}^{\operatorname{dim}\left(G_{0} / B_{0}\right)}(-1)^{i}\left[\Gamma_{i}\left(G_{0} / B_{0}, V\right)\right]
$$

then

$$
\left.C h \mathcal{E}_{0}(V)=\frac{1}{D_{0}} \sum_{w \in W} \varepsilon(w) w\left(e^{\rho_{0}} C h V\right)\right)
$$

by additivity of Euler characteristic.

Consider an injective resolution $0 \rightarrow R_{0} \rightarrow R_{1} \rightarrow \cdots$ of $C_{-\lambda}$ in the category of $B$ modules. By default it is an injective resolution in the category of $B_{0}$-modules. By definition $H^{i}\left(G / B, C_{-\lambda}\right)$ is given by the $i$-th cohomology of the complex

$$
0 \rightarrow H^{0}\left(G / B, R_{0}\right) \rightarrow H^{0}\left(G / B, R_{1}\right) \rightarrow \cdots .
$$

For any subalgebra $\mathfrak{l} \subset \mathfrak{g}$ containing $\mathfrak{h}$ we denote by $(\mathfrak{l}, \mathfrak{h})$-mod the category of $\mathfrak{l}$-modules semisimple over $\mathfrak{h}$. The universal enveloping algebra $U(\mathfrak{g})$ considered as $\left(\mathfrak{b}, \mathfrak{g}_{0}\right)$-bimodule has a finite filtration with the quotients $U(\mathfrak{b}) \otimes_{U\left(\mathfrak{b}_{0}\right)} S^{k}\left(\mathfrak{g}_{1} / \mathfrak{b}_{1}\right) \otimes U\left(\mathfrak{g}_{0}\right)$, with $k=1, \ldots, \operatorname{dim}\left(\mathfrak{g}_{1} / \mathfrak{b}_{1}\right)$. Since $R_{i}$ is injective in $(\mathfrak{b}, \mathfrak{h})$-mod, $\operatorname{Hom}_{U(\mathfrak{b})}\left(U(\mathfrak{g}), R_{i}\right)$ also has a filtration with quotients

$$
F_{i}^{k} \simeq \operatorname{Hom}_{U(\mathfrak{b})}\left(U(\mathfrak{b}) \otimes_{U\left(\mathfrak{b}_{0}\right)} S^{k}\left(\mathfrak{g}_{1} / \mathfrak{b}_{1}\right) \otimes U\left(\mathfrak{g}_{0}\right), R_{i}\right) \simeq \operatorname{Hom}_{U\left(\mathfrak{b}_{0}\right)}\left(U\left(\mathfrak{g}_{0}\right), R_{i} \otimes S^{k}\left(\mathfrak{g}_{1} / \mathfrak{b}_{1}\right)^{*}\right),
$$

where the latter isomorphism is an isomorphism of $\mathfrak{g}_{0}$-modules. Note that by construction $F_{i}^{k}$ are injective in $\left(\mathfrak{g}_{0}, \mathfrak{h}\right)$-mod, hence

$$
\operatorname{Hom}_{U(\mathfrak{b})}\left(U(\mathfrak{g}), R_{i}\right) \simeq \bigoplus_{k} F_{i}^{k}=\operatorname{Hom}_{U\left(\mathfrak{b}_{0}\right)}\left(U\left(\mathfrak{g}_{0}\right), R_{i} \otimes S\left(\mathfrak{g}_{1} / \mathfrak{b}_{1}\right)^{*}\right) .
$$

By (1) we have an isomorphism of $\mathfrak{g}_{0}$-modules

$$
H^{0}\left(G / B, R_{i}\right) \simeq H^{0}\left(G_{0} / B_{0}, R_{i} \otimes S\left(\mathfrak{g}_{1} / \mathfrak{b}_{1}\right)^{*}\right) .
$$


Since $R_{i} \otimes S\left(\mathfrak{g}_{1} / \mathfrak{b}_{1}\right)^{*}$ gives an injective resolution of $C_{-\lambda} \otimes S\left(\mathfrak{g}_{1} / \mathfrak{b}_{1}\right)^{*}$, the complex calculating $H^{i}\left(G_{0} / B_{0}, C_{-\lambda} \otimes S\left(\mathfrak{g}_{1} / \mathfrak{b}_{1}\right)^{*}\right)$ is the associated graded of (4). The Euler characteristics of both complexes are the same. Thus, we obtain

$$
C h \mathcal{E}(\lambda)=C h \mathcal{E}_{0}\left(C_{\lambda} \otimes S\left(\mathfrak{g}_{1} / \mathfrak{b}_{1}\right)\right) .
$$

The character of $C_{\lambda} \otimes S\left(\mathfrak{g}_{1} / \mathfrak{b}_{1}\right)$ is given by

$$
C h\left(C_{\lambda} \otimes S\left(\mathfrak{g}_{1} / \mathfrak{b}_{1}\right)\right)=e^{\lambda} \prod_{\alpha \in \Delta_{1}^{+}}\left(1+e^{-\alpha}\right)^{m_{\alpha}}=D_{1} e^{\lambda-\rho_{1}}
$$

Using (3) and $W$-invariance of $D_{1}$ one obtains (2).

Note that (2) implies the following

Lemma 2. - (a) For all $w \in W$, one has

$$
\mathcal{E}(\lambda)=\varepsilon(w) \mathcal{E}(w(\lambda+\rho)-\rho) .
$$

In particular, if $\langle\lambda+\rho, \check{\beta}\rangle=0$ for some even root $\beta$, then $\mathcal{E}(\lambda)=0$.

(b) The set

is linearly independent in $\mathcal{R}$.

$$
\left\{C h \mathcal{E}(\lambda), \lambda+\rho \in \Lambda^{+}\right\}
$$

Proof. - (a) follows immediately from (2). To prove (b) we observe that any $W$-orbit in $\Lambda$ with trivial stabilizer meets $\Lambda^{+}$in exactly one point. Hence the set $\left\{\sum_{w \in W} \varepsilon(w) e^{w(\lambda+\rho)}, \lambda+\right.$ $\left.\rho \in \Lambda^{+}\right\}$is linearly independent in $\mathcal{R}$. Therefore (2) implies (b).

We denote by $\mathcal{K}_{E}(\mathcal{F})$ the Grothendieck subgroup in $\mathcal{K}(\mathcal{F})$ generated by $\mathcal{E}(\lambda)$ for all $\lambda+\rho \in \Lambda^{+}$. By Lemma 1 in $[12] \mathcal{K}_{E}(\mathcal{F})$ is an ideal in $\mathcal{K}(\mathcal{F})$.

We continue with the following analogue of Bott's reciprocity result.

Proposition 1. - Let $M^{\mathfrak{h}}$ denote the set of $\mathfrak{h}$-invariants in an $\mathfrak{h}$-module $M$. Then, for any $B$-module $V$ and any dominant weight $\lambda$, we have

$$
\left[H^{i}(G / B, V): L_{\lambda}\right]=\operatorname{dim} H^{i}\left(\mathfrak{n}, P_{\lambda}^{*} \otimes V\right)^{\mathfrak{h}} .
$$

Proof. - For every $M \in \mathcal{F}$ we have

$$
\left[M: L_{\lambda}\right]=\operatorname{dim} \operatorname{Hom}_{\mathfrak{g}}\left(P_{\lambda}, M\right) .
$$

Consider an injective resolution $0 \rightarrow R_{0} \rightarrow R_{1} \rightarrow \cdots$ of $V$ in the category of $B$-modules. By definition $H^{i}(G / B, V)$ is given by the $i$-th cohomology of the complex (4). Since $\operatorname{Hom}_{G}\left(P_{\lambda}, \cdot\right)$ is an exact functor, $\operatorname{Hom}_{G}\left(P_{\lambda}, H^{i}(G / B, V)\right)$ is given by the $i$-th cohomology of the complex

$$
0 \rightarrow \operatorname{Hom}_{G}\left(P_{\lambda}, H^{0}\left(G / B, R_{0}\right)\right) \rightarrow \operatorname{Hom}_{G}\left(P_{\lambda}, H^{0}\left(G / B, R_{1}\right)\right) \rightarrow \cdots .
$$

Using Frobenius reciprocity we have

$$
\operatorname{Hom}_{G}\left(P_{\lambda}, H^{0}\left(G / B, R_{j}\right)\right)=\operatorname{Hom}_{B}\left(P_{\lambda}, R_{j}\right) .
$$

Thus, we obtain that

$$
\operatorname{Hom}_{G}\left(P_{\lambda}, H^{i}(G / B, V)\right)=\operatorname{Ext}_{B}^{i}\left(P_{\lambda}, V\right) \text {. }
$$

Now we recall that $B$ is the semidirect product of the torus $H$ and the unipotent supergroup $N$. Therefore it is easy to see that

$$
\operatorname{Ext}_{B}^{i}\left(P_{\lambda}, V\right)=\operatorname{Ext}_{\mathfrak{n}}^{i}\left(\left(P_{\lambda}, V\right)^{\mathfrak{h}}=H^{i}\left(\mathfrak{n}, P_{\lambda}^{*} \otimes V\right)^{\mathfrak{h}} .\right.
$$


By dualizing we obtain the following corollary.

Corollary 1. - For any weight $\nu$ and any dominant weight $\lambda$, we have

$$
\left[\Gamma_{i}\left(G / B, C_{\nu}\right): L_{\lambda}\right]=\operatorname{dim} H^{i}\left(\mathfrak{n}, P_{\lambda} \otimes C_{-\nu}\right)^{\mathfrak{h}} .
$$

Lemma 3. - Denote by $b(\nu, \lambda)$ the coefficients in the decomposition

$$
\mathcal{E}(\nu)=\sum_{\lambda} b(\nu, \lambda)\left[L_{\lambda}\right]
$$

One has:

(a) $b(\nu, \lambda)=\varepsilon(w) b(w(\nu+\rho)-\rho, \lambda)$.

(b) The coefficient $b(\nu, \lambda)$ is equal to the constant term in the formal expression $D^{-1} e^{-\nu-\rho} C h\left(P_{\lambda}\right)$.

(c) $C h P_{\lambda}=\sum_{\nu+\rho \in \Lambda^{+}} b(\nu, \lambda)\left(D \sum_{w \in W} \varepsilon(w) e^{w(\nu+\rho)}\right)$.

Proof. - (a) follows from Lemma 2 (a).

Corollary 1 implies

$$
b(\nu, \lambda)=\sum_{i}(-1)^{i} \operatorname{dim} H^{i}\left(\mathfrak{n}, P_{\lambda} \otimes C_{-\nu}\right)^{\mathfrak{h}} .
$$

Using the complex which computes the Lie superalgebra cohomology we obtain

$$
\begin{gathered}
\sum_{i}(-1)^{i} C h H^{i}\left(\mathfrak{n}, P_{\lambda} \otimes C_{-\nu}\right)=\sum_{i}(-1)^{i} C h\left(P_{\lambda} \otimes C_{-\nu} \otimes \Lambda^{i}\left(\mathfrak{n}^{*}\right)\right)= \\
=e^{-\nu} C h P_{\lambda} \sum_{i}(-1)^{i} C h \Lambda^{i}\left(\mathfrak{n}^{*}\right) .
\end{gathered}
$$

Since

$$
\sum_{i}(-1)^{i} C h \Lambda^{i}\left(\mathfrak{n}^{*}\right)=\frac{\prod_{\alpha \in \Delta_{0}^{+}}\left(1-e^{-\alpha}\right)}{\prod_{\alpha \in \Delta_{1}^{+}}\left(1+e^{-\alpha}\right)^{m_{\alpha}}}=D^{-1} e^{-\rho}
$$

we obtain (b).

Finally, (b) implies that for some finite subset $S \subset \Lambda$

$$
C h P_{\lambda}=\sum_{\nu \in S} b(\nu, \lambda) D e^{\nu+\rho} .
$$

Using (a) it can be rewritten in the form

$$
C h P_{\lambda}=\sum_{\nu+\rho \in \Lambda^{+} \cap S} b(\nu, \lambda)\left(D \sum_{w \in W} \varepsilon(w) e^{w(\nu+\rho)}\right) .
$$

Theorem 1. - We have the following identity in $\mathcal{K}(\mathcal{F})$

$$
\left[P_{\lambda}\right]=\sum_{\mu+\rho \in \Lambda^{+}} a(\lambda, \mu) \mathcal{E}(\mu) .
$$

Moreover, the following analogue of $B G G$ reciprocity holds

$$
a(\lambda, \mu)=b(\mu, \lambda) .
$$


Proof. - The statement follows from Lemma 3 (c) and Lemma 1.

Let $\mathcal{K}_{P}(\mathcal{F})$ be the subgroup in $\mathcal{K}(\mathcal{F})$ generated by classes of all projective modules. Since a tensor product of a projective module and any module is projective, $\mathcal{K}_{P}(\mathcal{F})$ is an ideal in $\mathcal{K}(\mathcal{F})$. Recall that $\mathcal{K}_{E}(\mathcal{F})$ is the subgroup in $\mathcal{K}(\mathcal{F})$ generated by the Euler characteristics. We have $\mathcal{K}_{P}(\mathcal{F}) \subset \mathcal{K}_{E}(\mathcal{F}) \subset \mathcal{K}(\mathcal{F})$. Then $b(\lambda, \mu)$ express the basis of of $\mathcal{K}_{E}(\mathcal{F})$ in terms of the basis of $\mathcal{K}(\mathcal{F})$ and $a(\nu, \lambda)$ express the basis of of $\mathcal{K}_{P}(\mathcal{F})$ in terms of the basis of $\mathcal{K}_{E}(\mathcal{F})$. For two dominant weights $\mu$ and $\nu$ we have

$$
\left[P_{\nu}: L_{\mu}\right]=\sum_{\lambda+\rho \in \Lambda^{+}} a(\nu, \lambda) b(\lambda, \mu)
$$

Example. Let $G=G L(m, n)$ and $B$ be the subgroup of upper triangular matrices. Then $\Lambda^{+}-\rho$ coincides with the set of dominant weights. Moreover, it is well-known (see for example [22]) that for any $\lambda \in \Lambda^{+}, \Gamma_{i}\left(G / B, C_{\lambda}\right)=0$ if $i>0$. Moreover,

$$
\Gamma_{0}\left(G / B, C_{\lambda}\right) \simeq K_{\lambda}:=U(\mathfrak{g}) \otimes_{U\left(\mathfrak{g}^{+}\right)} L_{\lambda}^{0},
$$

where $\mathfrak{g}^{+}=\mathfrak{g}_{0}+\mathfrak{b}$ and $L_{\lambda}^{0}$ is the irreducible $\mathfrak{g}_{0}$-module of highest weight $\lambda$ with trivial action of $\mathfrak{b}_{1}$. The module $K_{\lambda}$ was first considered in [17] and is usually called a Kac module. It was proven in [30] that every indecomposable projective module $P_{\lambda}$ has a filtration by Kac modules $K_{\mu}$ and that the multiplicity of $K_{\mu}$ in $P_{\lambda}$ equals the multiplicity of $L_{\lambda}$ in $K_{\mu}$. A combinatorial algorithm for calculating $a(\lambda, \mu)$ in this case was obtained by Brundan, [4]. We will explain it in Section 4 after introducing weight diagrams.

\section{Classical supergroups $G L(m, n)$ and $S O S P(m, 2 n)$}

In this section we collect all necessary facts about roots and weights for the classical supergroups. So we assume that $G=G L(m, n)$ or $\operatorname{SOSP}(m, 2 n)$.

The lattice $\Lambda$ of all integral weights is

$$
\Lambda=\bigoplus_{i=1}^{m} \mathbb{Z} \varepsilon_{i} \oplus \bigoplus_{j=1}^{n} \mathbb{Z} \delta_{i} .
$$

We define a parity homomorphism $p: \Lambda \rightarrow \mathbb{Z}_{2}$ by $p\left(\varepsilon_{i}\right)=0, p\left(\delta_{j}\right)=1$ for all $1 \leq i \leq m$, $1 \leq j \leq n$. The invariant form on $\Lambda$ is given by

$$
\left(\varepsilon_{i}, \varepsilon_{j}\right)=\delta_{i j},\left(\varepsilon_{i}, \delta_{j}\right)=0,\left(\delta_{i}, \delta_{j}\right)=-\delta_{i j} .
$$

Now we recall the description of $\Delta$ (see [16]).

The even roots $\Delta_{0}$ of $\mathfrak{g l}(m, n)$ are all the vectors of the form $\varepsilon_{i}-\varepsilon_{j}$ and $\delta_{i}-\delta_{j}$ with $i \neq j$. The odd roots $\Delta_{1}$ of $\mathfrak{g l}(m, n)$ are all the vectors of the form $\varepsilon_{i}-\delta_{j}$ and $\delta_{i}-\varepsilon_{j}$.

The even roots $\Delta_{0}$ of $\mathfrak{o s p}(2 m, 2 n)$ are all the vectors of the form $\pm \varepsilon_{i} \pm \varepsilon_{j}, \pm \delta_{i} \pm \delta_{j}$ (the signs can be chosen independently) with $i \neq j$ and $\pm 2 \delta_{i}$. The odd roots $\Delta_{1}$ of $\mathfrak{o s p}(2 m, 2 n)$ are all the vectors of the form $\pm \varepsilon_{i} \pm \delta_{j}$.

The even roots $\Delta_{0}$ of $\mathfrak{o s p}(2 m+1,2 n)$ are all the vectors of the form $\pm \varepsilon_{i} \pm \varepsilon_{j}, \pm \delta_{i} \pm \delta_{j}$ with $i \neq j, \pm \varepsilon_{i}$ and $\pm 2 \delta_{i}$. The odd roots $\Delta_{1}$ of $\mathfrak{o s p}(2 m+1,2 n)$ are all the vectors of the form $\pm \varepsilon_{i} \pm \delta_{j}$ and $\pm \delta_{i}$.

Recall that not all Borel subalgebras in a Lie superalgebra are conjugate. If one fixes an even Borel subalgebra $\mathfrak{b}_{0} \supset \mathfrak{h}$, the Borel subalgebras containing $\mathfrak{b}_{0}$ are related by odd reflections, see [28]. The condition $\lambda+\rho \in \Lambda^{+}$is not necessary for the dominance of $\lambda$. In the case $\mathfrak{g}=\mathfrak{g l}(m, n)$ it is possible to choose a distinguished Borel subalgebra $\mathfrak{b}$ (see [16]) so that all simple roots of $\mathfrak{b}_{0}$ are simple in $\mathfrak{b}$ and then $\lambda+\rho \in \Lambda^{+}$for all dominant $\lambda$. 
For $\mathfrak{o s p}(m, 2 n)$ such choice is impossible. Usually people fix a Borel subalgebra that has maximal number of even simple roots. However, for this choice a dominant weight $\lambda$ may have large negative $\langle\lambda+\rho, \check{\beta}\rangle$. We choose a Borel subalgebra which has maximal number of odd simple roots. Then for a dominant $\lambda$ we have $\langle\lambda+\rho, \check{\beta}\rangle \geq 0$ for $\mathfrak{g}=\mathfrak{o s p}(2 m, 2 n)$ and $\langle\lambda+\rho, \check{\beta}\rangle \geq-1$ for $\mathfrak{g}=\mathfrak{o s p}(2 m+1,2 n)$. One can probably repeat all our calculations for the canonical Borel subalgebra but that requires different notion of a weight diagram.

From now on we fix a Borel subalgebra of $\mathfrak{g}$, we make the same choice as in [12]. Below is the list of the simple roots for our choice of Borel subalgebras.

- If $\mathfrak{g}=\mathfrak{g l}(m, n), m \geq n$, the simple roots are

$$
\varepsilon_{1}-\varepsilon_{2}, \varepsilon_{2}-\varepsilon_{3}, \ldots, \varepsilon_{m}-\delta_{1}, \delta_{1}-\delta_{2}, \ldots, \delta_{n-1}-\delta_{n} .
$$

Since we consider $\mathfrak{g l}(m, n)$ instead of $\mathfrak{s l}(m, n)$ we can shift $\rho$ in the direction $\varepsilon_{1}+\varepsilon_{2}+\ldots+$ $\varepsilon_{m}-\delta_{1}-\ldots-\delta_{n}$ without any impact on representation theory. So we set

$$
\rho=m \varepsilon_{1}+(m-1) \varepsilon_{2}+\ldots+\varepsilon_{m}-\delta_{1}+\ldots+(-n) \delta_{n} ;
$$

- If $\mathfrak{g}=\mathfrak{o s p}(2 m+1,2 n)$ and $m \geq n$, the simple roots are

$$
\begin{gathered}
\varepsilon_{1}-\varepsilon_{2}, \ldots, \varepsilon_{m-n+1}-\delta_{1}, \delta_{1}-\varepsilon_{m-n+2}, \ldots, \varepsilon_{m}-\delta_{n}, \delta_{n}, \\
\rho=-\frac{1}{2} \sum_{i=1}^{m} \varepsilon_{i}+\frac{1}{2} \sum_{j+1}^{n} \delta_{j}+\sum_{i=1}^{m-n}(m-n-i+1) \varepsilon_{i} ;
\end{gathered}
$$

- If $\mathfrak{g}=\mathfrak{o s p}(2 m+1,2 n)$ and $m<n$, the simple roots are

$$
\begin{gathered}
\delta_{1}-\delta_{2}, \ldots, \delta_{n-m}-\varepsilon_{1}, \varepsilon_{1}-\delta_{n-m+1}, \ldots, \varepsilon_{m}-\delta_{n}, \delta_{n}, \\
\rho=-\frac{1}{2} \sum_{i=1}^{m} \varepsilon_{i}+\frac{1}{2} \sum_{j+1}^{n} \delta_{j}+\sum_{j=1}^{n-m}(n-m-j) \delta_{j} ;
\end{gathered}
$$

- If $\mathfrak{g}=\mathfrak{o s p}(2 m, 2 n)$ and $m>n$, the simple roots are

$$
\begin{gathered}
\varepsilon_{1}-\varepsilon_{2}, \ldots, \varepsilon_{m-n}-\delta_{1}, \delta_{1}-\varepsilon_{m-n+1}, \ldots, \delta_{n}-\varepsilon_{m}, \delta_{n}+\varepsilon_{m}, \\
\rho=\sum_{i=1}^{m-n}(m-n-i) \varepsilon_{i}
\end{gathered}
$$

- If $\mathfrak{g}=\mathfrak{o s p}(2 m, 2 n)$ and $m \leq n$, the simple roots are

$$
\begin{gathered}
\delta_{1}-\delta_{2}, \ldots, \delta_{n-m+1}-\varepsilon_{1}, \varepsilon_{1}-\delta_{n-m+2}, \ldots, \delta_{n}-\varepsilon_{m}, \delta_{n}+\varepsilon_{m}, \\
\rho=\sum_{i=1}^{n-m}(n-m-i+1) \delta_{i} .
\end{gathered}
$$

Finally, we give a description of $\Lambda^{+}$. Let

$$
\lambda+\rho=a_{1} \varepsilon_{1}+\ldots+a_{m} \varepsilon_{m}+b_{1} \delta_{1}+\ldots+b_{n} \delta_{n} .
$$

Then $\lambda$ is integral iff $a_{i}, b_{j} \in \mathbb{Z}$ for $G=G L(m, n)$ or $S O S P(2 m, 2 n)$, and $a_{i}, b_{j} \in \frac{1}{2}+\mathbb{Z}$ for $G=\operatorname{SOSP}(2 m+1,2 n)$. Furthermore, $\lambda+\rho \in \Lambda^{+}$if

$$
\begin{gathered}
a_{1}>a_{2}>\ldots>a_{m}, b_{1}>b_{2}>\ldots>b_{n} \text { if } G=G L(m, n) ; \\
a_{1}>a_{2}>\ldots>a_{m} \geq \frac{1}{2}, b_{1}>b_{2}>\ldots>b_{n} \geq \frac{1}{2} \text { if } G=\operatorname{SOSP}(2 m+1,2 n) ;
\end{gathered}
$$




$$
a_{1}>a_{2}>\ldots>a_{m-1}>\left|a_{m}\right| \text { and } b_{1}>b_{2}>\ldots>b_{n}>0 \text {, if } G=\operatorname{SOSP}(2 m, 2 n) .
$$

Every $\lambda \in \Lambda^{+}-\rho$ is dominant. If $G=G L(m, n)$, the set of dominant weights coincides with $\Lambda^{+}-\rho$. In the orthosymplectic case we will formulate the condition of dominance in the next section.

\section{Weight DiAgrams}

We recall the definitions and notations for weight diagrams, introduced in [6] for $G L(m, n)$, and in [12] for $\operatorname{SOSP}(m, 2 n)$. Note that our notations slightly differ from those in [6], for translation see [21].

4.1. Abstract weight diagrams. Let $\mathbb{T} \subset \mathbb{R}$ be a discrete set, $X=\left(x_{1}, \ldots, x_{m}\right) \in \mathbb{T}^{m}$, $Y=\left(y_{1}, \ldots, y_{n}\right) \in \mathbb{T}^{n}$. A diagram $f_{X, Y}$ is a function defined on $\mathbb{T}$ whose values are multisets with elements $<,>, \times$ according to the following algorithm.

- Put the symbol $>$ in position $t$ for all $i$ such that $x_{i}=t$.

- Put the symbol $<$ in position $t$ for all $i$ such that $y_{i}=t$.

- If there are both $>$ and $<$ in the same position replace them by the symbol $\times$, repeat if possible.

Thus, $f_{X, Y}(t)$ may contain at most one of the two symbols $>,<$. We represent $f_{X, Y}$ by the picture with $\circ$ standing in position $t$ whenever $f(t)$ is an empty set.

4.2. Weight diagrams for $G=G L(m, n)$. Let $G=G L(m, n)$. Let $\lambda$ be a dominant integral weight such that

$$
\lambda+\rho=a_{1} \varepsilon_{1}+\ldots+a_{m} \varepsilon_{m}+b_{1} \delta_{1}+\ldots+b_{n} \delta_{n} .
$$

Set $\mathbb{T}=\mathbb{Z}$

$$
X_{\lambda}=\left(a_{1}, \ldots, a_{m}\right), Y_{\lambda}=\left(-b_{1}, \ldots,-b_{n}\right) .
$$

The diagram $f_{\lambda}=f_{X_{\lambda}, Y_{\lambda}}$ is called the weight diagram of $\lambda$.

A diagram is the weight diagram of some dominant weight if and only if $|f(t)| \leq 1$ since both sequences $a_{1}, \ldots, a_{m}$ and $b_{1}, \ldots, b_{m}$ are strictly decreasing and hence do not have repetitions.

Examples. Let $G=G L(3,3)$. Then $\rho=(3,2,1 \mid-1,-2,-3)$. If $\lambda=0$ is the highest weight of the trivial module then

$$
f_{\lambda}=(\cdots \circ \times \times \times \circ \ldots),
$$

with first $\times$ at $t=1, \ldots$ corresponding to $\circ$. If $\lambda=\varepsilon_{1}-\delta_{1}$ is the highest weight of the adjoint module, then $f_{\lambda}$ is obtained from the previous diagram by moving the right $\times$ one position to the right:

$$
f_{\lambda}=(\cdots \circ \times \times \circ \times \cdots) .
$$

Now let $G=G L(5,3)$. Then $\rho=(5,4,3,2,1 \mid-1,-2,-3)$. For the trivial module the weight diagram is

$$
\cdots \circ \times \times \times>>0 \ldots
$$

and for the adjoint module the corresponding diagram is

$$
\cdots \circ \times \times>\times \circ>0 \ldots
$$


4.3. Weight diagrams for $G=\operatorname{SOSP}(2 m, 2 n)$. Set $\mathbb{T}=\mathbb{Z}_{\geq 0}$. For a dominant weight $\lambda$ such that $\lambda+\rho=a_{1} \varepsilon_{1}+\ldots+a_{m} \varepsilon_{m}+b_{1} \delta_{1} \ldots+b_{n} \delta_{n}$ let

$$
X_{\lambda}=\left(a_{1}, \ldots, a_{m-1},\left|a_{m}\right|\right), Y_{\lambda}=\left(b_{1}, \ldots, b_{n}\right), f_{\lambda}=f_{X_{\lambda}, Y_{\lambda}} .
$$

The position 0 is called the fail position. If the tail position is empty we put $[+]$ or $[-]$ before the diagram if $a_{m}>0$ or $a_{m}<0$ respectively.

A diagram $f_{\lambda}$ is the weight diagram of a dominant $\lambda$ if and only if

- for any $t \neq 0, f_{\lambda}(t)$ is empty or a single element set;

- the multiset $f_{\lambda}(0)$ does not contain $<$, contains $>$ with multiplicity at most 1 (it may contain any number of $x$ ).

A diagram $f_{\lambda}$ and a weight $\lambda$ are called tailless if $f(0)$ does not contain $\times$. A weight $\lambda$ is tailless iff $\lambda+\rho \in \Lambda^{+}$.

Examples. Let $G=\operatorname{SOSP}(4,2)$, then $\rho=0$. If $f_{\lambda}$ is the weight diagram of the highest weight of the trivial representation, then

$$
f_{\lambda}=\left({ }_{x} \circ \ldots\right) \text {. }
$$

If $\lambda=\varepsilon_{1}+\delta_{1}$ is the highest weight of the adjoint module, then

$$
f_{\lambda}=(>\times \circ \ldots) \text {. }
$$

Now let us interpret weight diagrams in terms of [10]. Recall that the authors encode dominant weights with non-negative $a_{m}$ by $(n \mid m)$-hook partitions, see Lemma 6.8 in [10]. Let $\zeta$ be a $(n \mid m)$-hook partition and $\zeta^{\perp}$ denote the conjugate partition. Then

$$
b_{i}=\max \left(\zeta_{i}-m+n-i+1,0\right), a_{j}=\max \left(\zeta_{j}^{\perp}-n+m-j, 0\right),
$$

for $i \leq n, j \leq m$.

The weight diagram corresponding to $\zeta$ is tailless if and only if $\zeta$ contains the box with coordinates $(m, n)$. The length of the tail is the number of boxes with coordinates $(m-x, n-x)(0 \leq x<\min (m, n))$ which are not contained in $\zeta$.

4.4. Weight diagrams for $G=\operatorname{SOSP}(2 m+1,2 n)$. Let $\mathbb{T}=\frac{1}{2}+\mathbb{Z}_{\geq 0}$ and define $X_{\lambda}$, $Y_{\lambda}$ and $f_{\lambda}$ as in the case $\mathfrak{g}=\mathfrak{o s p}(2 m, 2 n)$. The dominance condition is equivalent to the following condition on a weight diagram $f$

- $f(t)$ is empty or a single element set for any $t \neq \frac{1}{2}$;

- $f\left(\frac{1}{2}\right)$ may contain at most one of $<$ or $>$ and any number of $\times$.

The position $\frac{1}{2}$ is called the tail position. It is possible that two dominant weights have the same weight diagram. That may happen if $f\left(\frac{1}{2}\right)$ does not contain $>$ or $<$ and has at least one $\times$. For example, the diagram with two $\times$ at $\frac{1}{2}$ corresponds to $\left(\frac{1}{2},-\frac{1}{2} \mid \frac{1}{2}, \frac{1}{2}\right)$ and to $\left(-\frac{1}{2},-\frac{1}{2} \mid \frac{1}{2}, \frac{1}{2}\right)$. So if the weight diagram has at least one $\times$ and no $<,>$ at the position $\frac{1}{2}$ we put an indicator (which we sometimes refer to as "sign") ( \pm ) before the weight diagram. Its value is + if the corresponding weight has the form

$$
\lambda+\rho=\left(a_{1}, \ldots, a_{m-s}, \frac{1}{2},-\frac{1}{2}, \ldots,-\frac{1}{2} \mid b_{1}, \ldots, b_{n-s}, \frac{1}{2}, \ldots, \frac{1}{2}\right),
$$

and - if the corresponding weight has the form

$$
\lambda+\rho=\left(a_{1}, \ldots, a_{m-s},-\frac{1}{2},-\frac{1}{2}, \ldots,-\frac{1}{2} \mid b_{1}, \ldots, b_{n-s}, \frac{1}{2}, \ldots, \frac{1}{2}\right),
$$

where $s$ is the number of crosses at the position $\frac{1}{2}$.

A weight $\lambda$ is tailless if $f_{\lambda}\left(\frac{1}{2}\right)$ has at most one symbol, and if this symbol is $\times$ the indicator is + . As in the case $G=\operatorname{SOSP}(2 m, 2 n)$ a weight is tailless iff $\lambda+\rho \in \Lambda^{+}$. 
Examples. Let $G=\operatorname{SOSP}(5,4)$. Then $\rho=\left(-\frac{1}{2},-\frac{1}{2} \mid \frac{1}{2}, \frac{1}{2}\right)$. The diagram of the trivial module has two $\times$ at the tail position, all other positions are empty and the indicator is $(-)$. The weight diagram of the standard module $\left(\lambda=\varepsilon_{1}\right)$ is the same with indicator $(+)$. The weight diagram corresponding to the adjoint module $\left(\lambda=\varepsilon_{1}+\delta_{1}\right)$ is

$$
\vec{x}<0 \ldots
$$

Let $G=\operatorname{SOSP}(5,6)$. Then $\rho=\left(-\frac{1}{2},-\frac{1}{2} \mid \frac{1}{2}, \frac{1}{2}, \frac{1}{2}\right)$. The trivial module has the diagram with two $\times$ and one $<$ at the tail position, all other positions are empty. The standard module $\left(\lambda=\delta_{1}\right)$ has the diagram

$$
(-)_{\times}^{\times}<0 \ldots,
$$

and the adjoint module $\left(\lambda=2 \delta_{1}\right)$ has the diagram

$$
(-)_{\times}^{\times} \circ<\circ \ldots
$$

Let us again explain the connection between weight diagrams and combinatorial data in [10]. Dominant weights are encoded by $(n \mid m)$-hook partitions, see Lemma 6.1 in [10]. Let $\zeta$ be a $(n \mid m)$-hook partition and $\zeta^{\perp}$ denote the conjugate partition. Then

$$
b_{i}=\max \left(\zeta_{i}-m+n-i+\frac{1}{2}, \frac{1}{2}\right), a_{j}=\max \left(\zeta_{j}^{\perp}-n+m-j+\frac{1}{2},-\frac{1}{2}\right),
$$

for $i \leq n, j \leq m$.

As in the previous case, the weight diagram corresponding to $\zeta$ is tailless if and only if $\zeta$ contains the box with coordinate $(m, n)$. The length of the tail is the number of boxes with coordinates $(m-x, n-x)(0 \leq x<\min (m, n))$ which are not contained in $\zeta$.

4.5. Tailless weights, degree of atypicality and blocks. Here we collect all facts about weight diagrams which are true for all classical supergroups.

First of all, tailless diagrams are exactly the diagrams of weights in $\Lambda^{+}-\rho$. Hence tailless weights parametrize the basis $\mathcal{E}(\mu)$ in the lattice $\mathcal{K}_{E}(\mathcal{F})$.

The number of $\times$ in the diagram is called the degree of atypicality of the corresponding weight. The symbols $>,<$ are called the core symbols of the diagram. The core of a weight $\lambda$ is the diagram $f_{\lambda}$ with all $\times$ and the sign indicator removed except the case when $G=\operatorname{SOSP}(2 m, 2 n)$ and there are no $\times$. As follows from [12] two weights admit the same central character (i.e. the corresponding simple modules belong to the same block) if the cores of the two weights are the same. Thus, if two weights have the same central character, then they have the same degree of atypicality. Hence the degree of atypicality of a central character $\chi$ is well defined. We denote it by at $(\chi)$. A weight ( resp. central character) is typical if its degree of atypicality is zero. If $\chi$ is typical, then $\mathcal{F}_{\chi}$ is semisimple and has only one simple object up to isomorphism.

Let $\lambda$ be dominant and tailless. Following Brundan and Stroppel [9] we define the cap diagram of $f_{\lambda}$ by the following rules. The left end of a cap is at a $\times$ and the right end is at an empty position. We start from the rightmost $\times$, make the cap by joining it to the next free position on the right (the end is not free any more), and then repeat for the next $\times$ to the left, and so on until there is no $\times$ left. There is no empty position under any cap. One can see that for each weight diagram there is a unique cap diagram.

Example. For the following weight diagram, 


$0 \quad \times \quad<\quad \times \quad 0 \quad 0$

the caps are the following:

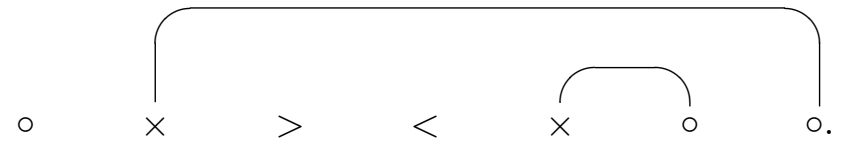

Denote by $\mathcal{P}(\lambda)$ the set of all the weights $\mu$ with weight diagram $f_{\mu}$ which can be obtained from $f_{\lambda}$ by flipping the labels at some caps. Note that the cardinality of $\mathcal{P}(\lambda)$ is $2^{k}$, where $k$ is the total number of $\times$.

If $G=G L(m, n)$, then all diagrams are tailless. The following result was proven in [4], see also (5.12) in [6].

Proposition 2. - Let $G=G L(m, n)$. Then

$$
[P(\lambda)]=\sum_{\mu \in \mathcal{P}(\lambda)} \mathcal{E}(\mu)
$$

\section{Categorification of $\mathfrak{g l}_{\infty / 2}$ in ORthosymplectic terms}

In this section we assume that $G=\operatorname{SOSP}(2 m+1,2 n)$. We will identify the lattice $\mathcal{K}_{E}(\mathcal{F})$ with a representation of $\mathfrak{g l}_{\infty / 2}$ and realize translation functors by Chevalley generators.

5.1. Representation of $\mathfrak{g l}_{\infty / 2}$. We denote by $\mathfrak{g l}_{\infty / 2}$ the infinite dimensional Lie algebra defined over $\mathbb{Z}$ with Dynkin diagram

$$
\circ-\circ-\circ-\circ \ldots
$$

and by $V$ its standard representation with basis $v_{1 / 2}, v_{3 / 2}, \ldots$ Let $w_{1 / 2}, w_{3 / 2}, \ldots$ be the basis in the costandard representation $V^{*}$ such that $\left\langle w_{i}, v_{j}\right\rangle=\delta_{i j}(-1)^{i+1 / 2}$. Let $E_{i, j}$ be the element of $\mathfrak{g l}_{\infty / 2}$ which acts on $V$ in the following way: $E_{i, j}\left(v_{k}\right)=\delta_{j k} v_{i}$ and on $V^{*}$ : $E_{i, j}\left(w_{k}\right)=(-1)^{i+j} \delta_{i k} w_{j}$.

Consider the representation (defined in the natural way) $\Lambda^{m}\left(V^{*}\right) \otimes \Lambda^{n}(V)$ of $\mathfrak{g l}_{\infty / 2}$. Then, if $\lambda$ is a tailless dominant weight for $\mathfrak{o s p}(2 m+1,2 n)$, such that $\lambda+\rho=\sum_{i=1}^{m} a_{i} \varepsilon_{i}+$ $\sum_{j=1}^{n} b_{j} \delta_{j}$ with $a_{i}, b_{j} \in 1 / 2+\mathbb{Z}$, we associate to $\lambda$ the following vector in $\Lambda^{m}\left(V^{*}\right) \otimes \Lambda^{n}(V)$ :

$$
x_{\lambda}:=w_{a_{1}} \wedge \ldots \wedge w_{a_{m}} \otimes v_{b_{1}} \wedge \ldots \wedge v_{b_{n}} .
$$

We denote the weight of the action of $\mathfrak{g l}_{\infty / 2}$ on $v_{i}$ by $\gamma_{i}$ (hence, the weight of the action

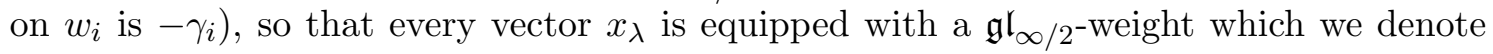
by $\gamma(\lambda)$.

Remark. - The weights with the same core have the same $\mathfrak{g l}_{\infty / 2}$-weight. Therefore for every pair of tailless dominant weights $\lambda, \mu$, one has

$$
\chi_{\lambda}=\chi_{\mu} \Leftrightarrow \gamma(\lambda)=\gamma(\mu) \text {. }
$$


Define the map $\varphi: \mathcal{K}_{E}(\mathcal{F}) \rightarrow \Lambda^{m}\left(V^{*}\right) \otimes \Lambda^{n}(V)$ by $\varphi(\mathcal{E}(\lambda))=x_{\lambda}$ for all tailless dominant $\lambda$. Obviously, $\varphi$ is an isomorphism of abelian groups.

5.2. Interpretation of the translation functors in this setting. Since the weight of the $\mathfrak{g l}_{\infty / 2}$-action encodes the central character, every block in $\mathcal{F}$ can be parametrized by a $\mathfrak{g l}_{\infty / 2}$-weight $\gamma$, hence $\mathcal{F}=\oplus_{\gamma} \mathcal{F}_{\gamma}$. The translation functors consist in tensoring with the standard module $E$ of $\mathfrak{o s p}(2 m+1,2 n)$ and then projecting in the appropriate block.

For any $M \in \mathcal{F}_{\gamma}, a \in \frac{1}{2}+\mathbb{Z}_{\geq 0}$, we set:

$$
\begin{aligned}
& T_{a, a+1}(M)=(M \otimes E)_{\gamma+\gamma_{a}-\gamma_{a+1}}, \\
& T_{a+1, a}(M)=(M \otimes E)_{\gamma+\gamma_{a+1}-\gamma_{a}} .
\end{aligned}
$$

The functors $T_{a, a+1}$ and $T_{a+1, a}$, being exact, induce linear operators in the Grothendieck group $\mathcal{K}(\mathcal{F})$, and we keep the same notations for them. Note also that

$\operatorname{Hom}_{\mathfrak{g}}\left(M, T_{a, a+1}(N)\right)=\operatorname{Hom}_{\mathfrak{g}}\left(T_{a+1, a}(M), N\right), \operatorname{Hom}_{\mathfrak{g}}\left(M, T_{a+1, a}(N)\right)=\operatorname{Hom}_{\mathfrak{g}}\left(T_{a, a+1}(M), N\right)$,

for any $M, N \in \mathcal{F}$. Hence $T_{a, a+1}$ and $T_{a+1, a}$ are adjoint.

It was shown in [12] (Lemma 1(b)and Corollary 1),

$$
\Gamma_{i}\left(G / B, C_{\lambda} \otimes E\right)=\Gamma_{i}\left(G / B, C_{\lambda}\right) \otimes E .
$$

Therefore $\mathcal{K}_{E}(\mathcal{F})$ is invariant under both $T_{a, a+1}$ and $T_{a+1, a}$.

Lemma 4. - For any $a \geq \frac{1}{2}$ one has

$$
\varphi \circ T_{a+1, a}=E_{a+1, a} \circ \varphi
$$

and

$$
\varphi \circ T_{a, a+1}=E_{a, a+1} \circ \varphi
$$

Proof. - Let $\gamma^{\prime}$ be a $\mathfrak{g l}_{\infty / 2^{-}}$-weight and let $\lambda$ be a tailless dominant $\mathfrak{o s p}(2 m+1,2 n)$-weight with $\gamma(\lambda)=\gamma$. Corollary 1 in [12] implies that

$$
\Gamma_{i}\left(G / B,\left(C_{\lambda} \otimes E\right)_{\Phi^{-1}\left(\gamma^{\prime}\right)}\right)=\left(\Gamma_{i}\left(G / B, C_{\lambda}\right) \otimes E\right)_{\gamma^{\prime}},
$$

where $\Phi^{-1}\left(\gamma^{\prime}\right)$ is the set of all weights $\mu$ such that $\gamma(\mu)=\gamma^{\prime}$.

The $\mathfrak{b}$-module $L_{\lambda}(\mathfrak{b}) \otimes E$ has a filtration by all the $C_{\mu}$ with $\mu=\lambda \pm \varepsilon_{j}, \lambda \pm \delta_{k}, \lambda$. Since the Euler characteristic of the associated graded sheaf coincides with the Euler characteristic of the original sheaf, we obtain

$$
T_{a, a+1}(\mathcal{E}(\lambda))=\sum_{\mu} \mathcal{E}(\mu)
$$

where the sum is taken over all $\mu=\lambda \pm \varepsilon_{j}, \lambda \pm \delta_{k}$ such that $\gamma(\mu)=\gamma(\lambda)+\gamma_{a}-\gamma_{a+1}$.

By direct inspection if $\mu$ is not dominant, then $(\mu+\rho, \beta)=0$ for some even root $\beta$ and therefore by $(2) \mathcal{E}(\mu)=0$. Hence in fact only dominant $\mu$ contribute in the summation. To find all such $\mu$ we use the weight diagram $f_{\lambda}$ of $\lambda$, and we only have to look at the positions $a$ and $a+1$ in $f_{\lambda}$. Here is a table of the different $f_{\mu}$ which can occur (see figure 1 ). Note that since only tailless weights appear, if the left position is $\frac{1}{2}$, and there is a $\times$ then the sign before it should be $(+)$ and we omit it for simplicity of notation. 


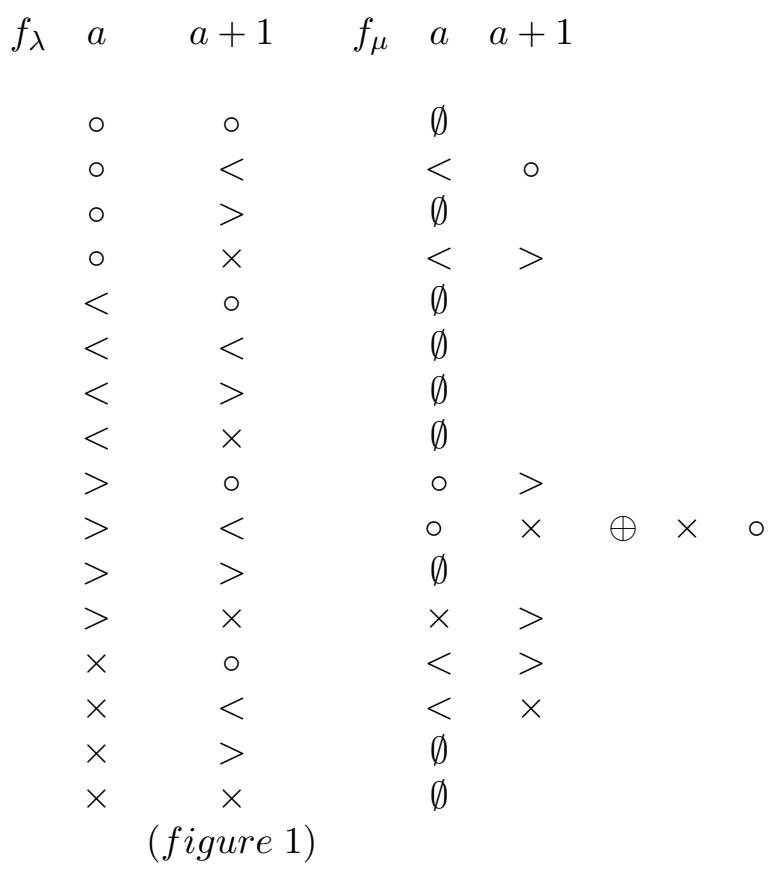

Now we conclude, noticing that

$$
T_{a, a+1}\left(\mathcal{E}_{\lambda}\right)=\sum_{\mu+\rho \in \Lambda^{+}} \mathcal{E}_{\mu}
$$

with the weights $\mu$ we computed, and since

$$
E_{a, a+1}\left(x_{\lambda}\right)=\sum_{\mu+\rho \in \Lambda^{+}} x_{\mu}
$$

by construction, we have proved the first identity. The proof of the second identity is similar and we leave it to the reader.

Remark. - Thus, the isomorphism $\varphi: \mathcal{K}_{E}(\mathcal{F}) \rightarrow \Lambda^{m}\left(V^{*}\right) \otimes \Lambda^{n}(V)$ is an isomorphism of $\mathfrak{g l}_{\infty / 2}$-modules.

5.3. The switch functor. In [12] we constructed a certain functor in the category $\mathcal{F}$ of $\mathfrak{o s p}(2 m+1,2 n)$-modules. When applied to a simple module, it switches the sign of the corresponding weight diagram. By this reason we call it the switch functor. It is very important for calculation of $a(\lambda, \mu)$ since it increases the length of the tail of a diagram if the sign changes from $(+)$ to $(-)$. In particular, this functor can map $L_{\lambda}$ with $\lambda+\rho \in \Lambda^{+}$ to $L_{\mu}$ with $\mu+\rho \notin \Lambda^{+}$. The functors $T_{a, a \pm 1}$ do not give such an option.

Recall that the switch functor $s w: \mathcal{F}_{\gamma} \rightarrow \mathcal{F}_{\gamma}$ is defined by

$$
s w(M)=(M \otimes E)_{\gamma} .
$$

We also denote by $s w$ the corresponding linear operator in $\mathcal{K}(\mathcal{F})$.

The following lemma explains how sw acts on $\mathcal{E}(\lambda)$.

Lemma 5. - Let $\lambda$ be a tailless dominant weight. If $f_{\lambda}$ has an empty tail position, then $s w(\mathcal{E}(\lambda))=\mathcal{E}(\lambda)$.

If $f_{\lambda}$ has $\times$ at the tail position, then $s w(\mathcal{E}(\lambda))=-\mathcal{E}(\lambda)$. 
If $f_{\lambda}$ has $>$ or $<$ at the tail position, then $s w(\mathcal{E}(\lambda))=0$.

Proof. - We use the same idea as in the proof of Lemma 4. The weights of $E$ are 0 , $\pm \varepsilon_{i}, i=1, \ldots, m$ and $\pm \delta_{j}, j=1, \ldots, n$ By Lemma 1 in [12] and we have the following identity in the Grothendieck ring:

$$
\mathcal{E}(\lambda)[E]=\sum_{\nu} \mathcal{E}(\lambda+\nu)
$$

where $\nu$ runs the set of weights of $E$. When we apply the switch functor we just pick up the summands which have the same central character as $\lambda$. Thus, as follows from Corollary 1 in [12]

$$
s w(\mathcal{E}(\lambda))=\mathcal{E}(\lambda)+\sum_{\mu} \mathcal{E}(\mu)
$$

where the summation is over all $\mu=\lambda \pm \varepsilon_{j}, \lambda \pm \delta_{k}$ such that $\gamma(\mu)=\gamma(\lambda)$.

Looking at the weight diagram $f_{\lambda}$ we see that the number of summands in the latter formula depends only on the tail position. Indeed, if $a_{i}>\frac{1}{2}$ (resp. $b_{j}>\frac{1}{2}$ ) the core of $f_{\lambda \pm \varepsilon_{i}}$ (resp. of $f_{\lambda \pm \delta_{j}}$ ) is different from the core of $f_{\lambda}$.

If $f_{\lambda}$ has empty tail position, then the only weight appearing in the sum is $\lambda$ itself.

If $f_{\lambda}$ has $>$ at the tail position, then the weights appearing in the sum are $\lambda$ and $\lambda-\varepsilon_{m}$. But $\lambda-\varepsilon_{m}=s(\lambda+\rho)-\rho$ where $s$ is the reflection with respect to the root $\varepsilon_{m}$. Hence by (2) $\mathcal{E}\left(\lambda-\varepsilon_{m}\right)=-\mathcal{E}(\lambda)$ and $s w(\mathcal{E}(\lambda))=0$.

Similarly, if $f_{\lambda}$ has $<$ at the tail position,

$$
s w(\mathcal{E}(\lambda))=\mathcal{E}(\lambda)+\mathcal{E}\left(\lambda-\delta_{n}\right)=0 .
$$

Finally, if $f_{\lambda}$ has $\times$ at the tail position, then

$$
s w(\mathcal{E}(\lambda))=\mathcal{E}(\lambda)+\mathcal{E}\left(\lambda-\varepsilon_{m}\right)+\mathcal{E}\left(\lambda-\delta_{n}\right)=-\mathcal{E}(\lambda) .
$$

\section{Translation FUnCtors aCtion ON SIMPle AND PROJECTIVE MOdUlES}

In this section $G=S O S P(2 m+1,2 n)$. We study here the action of translation functors on simple and indecomposable projective modules. The combinatorial statements of this section are about all dominant weights (possibly with tail).

\subsection{Functors $T_{a, a+1}$ and $T_{a+1, a}$.}

Lemma 6. - Let $L_{\lambda}, L_{\nu} \in \mathcal{F}, \nu=\lambda-\alpha$ for some isotropic positive root $\alpha=\varepsilon_{i}+\delta_{j}$ such that $(\lambda+\rho, \alpha)=0$. Then

$$
\left[\Gamma_{0}\left(G / B, C_{\lambda}\right): L_{\lambda}\right]=1, \quad\left[\Gamma_{0}\left(G / B, C_{\lambda}\right): L_{\nu}\right] \geq 1
$$

Proof. - By Lemma 2 in [12] $\Gamma_{0}\left(G / B, C_{\lambda}\right)$ is the maximal finite dimensional quotient of the Verma module with highest weight $\lambda$. Hence $\left[\Gamma_{0}\left(G / B, C_{\lambda}\right): L_{\lambda}\right]=1$. Propositions 2 and 5 in [12] imply that for some parabolic subgroup $P$ containing $B$ we have $\left[\Gamma_{0}\left(G / P, L_{\lambda}(P)\right)\right.$ : $\left.L_{\nu}\right]=1$, where $L_{\lambda}(P)$ is a simple $P$-module with highest weight $\lambda$. By Lemma 2 in [12] there is a surjective homomorphism

$$
\Gamma_{0}\left(G / B, C_{\lambda}\right) \rightarrow \Gamma_{0}\left(G / P, L_{\lambda}(P)\right)
$$

Hence the statement. 
In the case of $G L(m, n)$ the following result first appeared in [24], see also Lemma 3.4 in $[8]$.

Lemma 7. - Let $\chi$ and $\theta$ be two distinct central characters such that at $(\chi) \geq$ at $(\theta)$. Denote by $T$ the translation functor which maps $\mathcal{F}_{\chi}$ to $\mathcal{F}_{\theta}$. Then, if some dominant weight $\lambda$ (resp. $\left.\lambda_{1}, \lambda_{2}\right)$ has central character $\chi$, then $T\left(L_{\lambda}\right)$ is either a simple module of $\mathcal{F}_{\theta}$ or zero.

Moreover, if $T\left(L_{\lambda_{1}}\right)=T\left(L_{\lambda_{2}}\right) \neq 0$, then $\lambda_{1}=\lambda_{2}$.

Proof. - All $\mathfrak{b}$-singular vectors in $L_{\lambda} \otimes E$ have weights of the form $\lambda \pm \varepsilon_{j}, \lambda, \lambda \pm \delta_{k}$. At most one of those has the central character $\theta$, as one can figure out writing the weight diagrams. Hence the first statement.

Assume now that $T\left(L_{\lambda_{1}}\right)=T\left(L_{\lambda_{2}}\right)=L_{\mu}$, then $\lambda_{1}-\lambda_{2}$ is an isotropic root, a multiple of an even root, or zero.

Case 1: $\lambda_{1}-\lambda_{2}$ is a multiple of an even root say $\beta$ ( one has $\lambda_{1}-\lambda_{2}= \pm 2 \varepsilon_{i}, \varepsilon_{i}-\varepsilon_{j}, \pm 2 \delta_{i}$ or $\left.\delta_{i}-\delta_{j}\right)$ and then $\lambda_{1}+\rho=s_{\beta}\left(\lambda_{2}+\rho\right)$, where $s_{\beta}$ is the reflection with respect to the root $\beta$. Hence $\lambda_{1}$ and $\lambda_{2}$ cannot be both dominant except if $\left(\lambda_{1}+\rho, \beta\right)=0$, in which case $\lambda_{1}=\lambda_{2}$.

Case 2: $\lambda_{1}-\lambda_{2}$ is an isotropic root $\alpha= \pm\left(\varepsilon_{i}+\delta_{j}\right)$, which we can assume to be a positive root. As follows from Lemma 6

$$
\left[\Gamma_{0}\left(G / B, C_{\lambda_{1}}\right): L_{\lambda_{1}}\right]=1\left[\Gamma_{0}\left(G / B, C_{\lambda_{1}}\right): L_{\lambda_{2}}\right] \geq 1 .
$$

By Corollary 1 in [12] (see also (6))

$$
T\left(\Gamma_{0}\left(G / B, C_{\lambda_{1}}\right)\right)=\Gamma_{0}\left(G / B, C_{\mu}\right) .
$$

As $\left[\Gamma_{0}\left(G / B, C_{\mu}\right): L_{\mu}\right]=1$, either $T\left(L_{\lambda_{1}}\right)$ or $T\left(L_{\lambda_{2}}\right)$ is zero.

Lemma 8. - Let $\chi$ and $\theta$ be two distinct central characters such that at $(\chi) \leq a t(\theta)$. Then the translation functor $T$ which maps $\mathcal{F}_{\chi}$ to $\mathcal{F}_{\theta}$ maps projective indecomposable modules (PIMs for short) to PIMs or to zero.

Proof. - Let $\lambda$ be a dominant weight with central character $\chi$. Since $T\left(P_{\lambda}\right)$ is a projective module in $\mathcal{F}_{\theta}$, it is sufficient to show that

$$
\operatorname{Hom}_{\mathfrak{g}}\left(T\left(P_{\lambda}\right), L_{\mu}\right)=\mathbb{C}
$$

for at most one dominant weight $\mu$, and is zero otherwise.

Let us denote by $T^{*}$ the adjoint functor of $T$, which is the translation functor mapping $\mathcal{F}_{\theta}$ to $\mathcal{F}_{\chi}$; one has

$$
\operatorname{Hom}_{\mathfrak{g}}\left(T\left(P_{\lambda}\right), L_{\mu}\right)=\operatorname{Hom}_{\mathfrak{g}}\left(P_{\lambda}, T^{*}\left(L_{\mu}\right)\right)
$$

and by the Lemma 7 , the statement follows.

\subsection{The action of the switch functor on simple modules and PIMs.}

Lemma 9. - Let $\lambda$ be an integral dominant weight.

If $f_{\lambda}$ has the empty tail position, then $\operatorname{sw}\left(L_{\lambda}\right)=L_{\lambda}$.

If $f_{\lambda}$ has $>$ or $<$ at the tail position, then $s w\left(L_{\lambda}\right)=0$.

If $f_{\lambda}$ has several $\times$ at the tail position and no core symbols, then $s w\left(L_{\lambda}\right)=L_{\mu}$, where $f_{\mu}$ is obtained from $f_{\lambda}$ by change of sign.

The action of the switch functor on PIMs is given by the same rule. 
Proof. - The first assertion easily follows from

$$
s w\left(\Gamma_{0}\left(G / B, C_{\lambda}\right)\right)=\Gamma_{0}\left(G / B, C_{\lambda}\right) .
$$

To prove the second assertion choose the parabolic subalgebra $\mathfrak{q} \subset \mathfrak{g}$ with semisimple part $\mathfrak{o s p}(2 k+3,2 k)$ if $f_{\lambda}$ has $k \times$ and $>$ at the tail position and with semisimple part $\mathfrak{o s p}(2 k+1,2 k+2)$ if $f_{\lambda}$ has $k \times$ and $<$ at the tail position. Then $C_{\lambda}$ has a natural $\mathfrak{q}$-module structure and $L_{\lambda}$ is a quotient of the parabolically induced module $S_{\lambda}=U(\mathfrak{g}) \otimes_{U(\mathfrak{q})} C_{\lambda}$. It is easy to check that

$$
s w\left(S_{\lambda}\right)=\left(U(\mathfrak{g}) \otimes_{U(\mathfrak{q})}\left(C_{\lambda} \otimes E\right)\right)_{\gamma(\lambda)}=0 .
$$

Hence $s w\left(L_{\lambda}\right)=0$.

Now let $f_{\lambda}$ have $k \times$-s at the tail position and no core symbols. Let $\mathfrak{q}$ be the parabolic subalgebra with semisimple part $\mathfrak{o s p}(2 k+1,2 k)$. If the sign of $f_{\lambda}$ is - then $L_{\lambda}$ is a quotient of $S_{\lambda}$. On the other hand,

$$
S_{\lambda}^{\prime}:=s w\left(S_{\lambda}\right)=U(\mathfrak{g}) \otimes_{U(\mathfrak{q})}\left(C_{\lambda} \otimes E^{\prime}\right),
$$

where $E^{\prime}$ is the standard module over semisimple part of $\mathfrak{q}$. The unique simple quotient of $S_{\lambda}^{\prime}$ is isomorphic to $L_{\mu}$, where $f_{\mu}$ is obtained from $f_{\lambda}$ by change of sign. Since the application of the switch functor to any other simple subquotient of $S_{\lambda}$ can not produce $L_{\mu}$ we have $s w\left(L_{\lambda}\right)=L_{\mu}$. If the sign of $f_{\lambda}$ is + , the assertion follows similarly from the fact that $s w\left(S_{\lambda}^{\prime}\right)=S_{\lambda}$.

Finally, the statement about PIMs follows by duality.

6.3. Elementary changes. The main idea in calculation of $a(\lambda, \mu)$ is to start with a typical weight $\lambda$, for which the problem is trivial, and move to an arbitrary weight by use of translation functors. Each time we want to map a PIM to a PIM (see Lemma 10). At the level of weight diagrams such functors correspond to elementary changes which we define by the list.

- The change of the sign $(+)$ and $(-)$ in front of the diagram.

- An elementary change which involves positions $a$ and $a+1$ with $a>\frac{1}{2}$ :

a) $\ldots \times \circ \ldots \rightarrow \ldots><\ldots$ (decreases the degree of atypicality by 1$)$,

b) $\ldots<\circ \ldots \leftrightarrow \ldots \circ<\ldots$ (doesn't change the degree of atypicality),

c) $\ldots>\circ \ldots \leftrightarrow \ldots \circ>\ldots$ (doesn't change the degree of atypicality),

- An elementary change which involves positions $\frac{1}{2}$ and $\frac{3}{2}$

at) $(+) x^{k} \circ \ldots \rightarrow_{x^{k-1}}^{>}<\ldots$ (decreases the degree of atypicality by 1$)$,

bt) $x^{k} \circ \ldots \leftrightarrow(-) x^{k}>\ldots$ (doesn't change the degree of atypicality),

ct) $\underset{x^{k}}{<} \circ \ldots \leftrightarrow(-) \times^{k}<\ldots$ (doesn't change the degree of atypicality),

The $\times^{k}$ sign indicates that there are $k \times$ at the tail position and $\leftrightarrow$ means that we can go in either direction.

It is convenient to divide elementary changes in two groups: ones that do not involve the tail position and ones that involve the tail position. We call the latter the tail elementary changes.

Lemma 10. - If $\left(f_{\lambda}, f_{\mu}\right)$ is a pair of weight diagrams of the list a),.., ct), then for a suitable choice of a translation functor $T$ we have $T\left(L_{\lambda}\right)=L_{\mu}$ and $T^{*}\left(P_{\mu}\right)=P_{\lambda}$.

Proof. - In the cases b),c),bt) and ct), we already know ([12] Section 6) that $T$ is an equivalence of the corresponding blocks, thus we have nothing to prove. 
It remains to prove the statement for a pair $\left(f_{\lambda}, f_{\mu}\right)$ in the cases a)and at).

We have $\mu=\lambda+\delta_{a+1}$. It is easy to see that among the weights $\lambda \pm \varepsilon_{i}, \lambda \pm \delta_{j}$ only $\mu$ has weight $\gamma(\mu)$. Therefore by Corollary 1 in [12] we have

$$
T\left(\Gamma_{0}\left(G / B, C_{\lambda}\right)\right)=\Gamma_{0}\left(G / B, C_{\mu}\right) .
$$

The multiplicity of $L_{\lambda}$ in $\Gamma_{0}\left(G / B, C_{\lambda}\right)$ is 1 as well as the multiplicity of $L_{\mu}$ in $\Gamma_{0}\left(G / B, C_{\mu}\right)$. If $L_{\nu}$ is a simple subquotient of $\Gamma_{0}\left(G / B, C_{\lambda}\right)$ and $\nu \neq \mu$, then $\nu<\mu$ and by Lemma 7 $T\left(L_{\nu}\right) \neq L_{\mu}$. Therefore (7) implies $T\left(L_{\lambda}\right)=L_{\mu}$.

Lemma 11. - Let $\lambda$ be any dominant weight with atypicality degree $k>0$. Then one can find a dominant typical weight $\mu$ such that there exist a sequence of weights $\mu=$ $\mu_{1}, \mu_{2}, \ldots, \mu_{r}=\lambda$ and translation functors $T_{1}, \ldots, T_{r-1}$ such that $P_{\mu_{i}}=T_{i-1}\left(P_{\mu_{i-1}}\right)$, and at $\left(\mu_{i}\right) \leq a t\left(\mu_{i+1}\right)$.

Proof. - Due to the previous Lemma we have to check that $f_{\lambda}$ can be transformed to a typical $f_{\mu}$ by elementary changes.

We prove the statement by induction on degree of atypicality of $\lambda$. Let $t$ be the position of the rightmost $\times$ in the weight diagram $f_{\lambda}$. Assume first that $t \neq \frac{1}{2}$. If the position $t+1$ is empty we can use elementary change a) to decrease the atypicality degree of $\lambda$. If the position $t+1$ is occupied by a core symbol, we can use elementary changes of type b) and c) to move all core symbols in positions $t+1, t+2, \ldots$ to the right. The diagram obtained in this way will have the position $t+1$ empty and now we can decrease the degree of atypicality using elementary change a).

Now let $t=\frac{1}{2}$. That means $f_{\lambda}$ has only core symbols outside the tail position. Using elementary changes of type b) and c) we can transform $f_{\lambda}$ to the diagram that has an empty position $\frac{3}{2}$. Hence without loss of generality we may assume that the position $\frac{3}{2}$ in $f_{\lambda}$ is empty. Now we are going consider 3 different cases.

If all symbols at the tail positions are $\times$ and the sign is + , we apply elementary change at) to decrease the degree of atypicality.

If all symbols at the tail positions are $\times$ and the sign is - , we apply the switch functor to $f_{\lambda}$ and reduce the situation to the previous case.

If the tail position contains a core symbol, we apply elementary change bt) or ct) and reduce the situation to the previous case.

Thus, the statement follows by induction.

\section{The action of translation Functors in the CASE $S O S P(2 m, 2 n)$}

In this section $G=S O S P(2 m, 2 n)$. There is an involutive automorphism $\sigma$ preserving the maximal torus $H$ which acts on $\Lambda$ by the formula

$$
\sigma\left(\varepsilon_{m}\right)=-\varepsilon_{m}, \sigma\left(\varepsilon_{i}\right)=\varepsilon_{i} \text { if } i \neq m \sigma\left(\delta_{j}\right)=\delta_{j} .
$$

The induced action of $\sigma$ on the weight diagrams is the change of the sign if the diagram has a sign, otherwise $\sigma$ preserves the weight diagram.

The action of $\sigma$ on $\mathcal{F}$ preserves blocks except the case when $\chi$ is typical and the diagram has an empty tail position. In the latter case $\sigma$ permutes two blocks. If $M \in \mathcal{F}$ we denote by $M^{\sigma}$ the twist of $M$ by $\sigma$.

The induced action of $\sigma$ on the Grothendieck ring $\mathcal{K}(\mathcal{F})$ has two eigenspaces $\mathcal{K}(\mathcal{F})^{ \pm}$ with eigenvalues \pm 1 . So we have the following decompositions

$$
\mathcal{K}(\mathcal{F})=\mathcal{K}(\mathcal{F})^{+} \oplus \mathcal{K}(\mathcal{F})^{-}
$$


and

$$
\mathcal{K}_{E}(\mathcal{F})=\mathcal{K}_{E}(\mathcal{F})^{+} \oplus \mathcal{K}_{E}(\mathcal{F})^{-}, \mathcal{K}_{P}(\mathcal{F})=\mathcal{K}_{P}(\mathcal{F})^{+} \oplus \mathcal{K}_{P}(\mathcal{F})^{-} .
$$

Note that the translation functors preserve those splittings.

7.1. Categorification of $\mathfrak{g l}_{\infty}$. As in Section 5 we identify the lattice $\mathcal{K}_{E}(\mathcal{F})$ with a representation of $\mathfrak{g l}_{\infty}$ and realize translation functors by linear operators corresponding to Chevalley generators in this representation. $B y \mathfrak{g l}_{\infty}$ we understand the Lie algebra with Dynkin diagram

$$
\ldots-0-0-0-0 \ldots
$$

and by $U$ its standard representation with basis $u_{i}$ with $i \in \mathbb{Z}$. Let $E_{i, j}$ be the element of $\mathfrak{g l}_{\infty}$ which acts on $V$ the following way: $E_{i, j}\left(v_{k}\right)=\delta_{j k} v_{i}$. Denote the weight of $v_{a}$ by $\gamma_{a}$ for $a>0$ and by $-\gamma_{-a}$ for $a<0$. Set $\gamma_{0}=0$. Let $U \leq 0$ be the span of $u_{i}$ for all $i \leq 0, U^{+}$ and $U^{-}$be the span of $u_{i}$ for $i>0$ and $i<0$ respectively. Set

$$
\begin{gathered}
X^{+}=\Lambda^{m}\left(U^{\leq 0}\right) \otimes \Lambda^{n}\left(U^{+}\right), \\
X^{-}=\Lambda^{m}\left(U^{-}\right) \otimes \Lambda^{n}\left(U^{+}\right) .
\end{gathered}
$$

Finally set

$$
F_{i, j}=(-1)^{i+j+1}\left(E_{i, j}+E_{-j,-i}\right), \text { for } i, j>0 ; F_{i, 0}=2 E_{0,-i}, F_{0, i}=E_{-i, 0} .
$$

Those elements generate the Lie algebra $\mathfrak{s l}_{\infty / 2} \oplus \mathfrak{s l}_{\infty / 2}$ inside $\mathfrak{g l}_{\infty}$.

Let $\lambda+\rho \in \Lambda^{+}$(i.e. $\lambda$ is tailless) be such that $\lambda+\rho=\sum_{i=1}^{m} a_{i} \varepsilon_{i}+\sum_{j=1}^{n} b_{j} \delta_{j}$ with $a_{i} \in \mathbb{Z}_{\geq 0}, b_{j} \in \mathbb{Z}_{>0}$, we associate to $\lambda$ the vector

$$
x_{\lambda}:=u_{-a_{m}} \wedge \ldots \wedge u_{-a_{1}} \otimes u_{b_{1}} \wedge \ldots \wedge u_{b_{n}} .
$$

The weight of $x_{\lambda}$ and the translation functors $T_{a, a+1}$ and $T_{a+1, a}$ (for $a \in \mathbb{Z}_{>0}$ ) are defined as in Section 5. In addition we define the translation functors $T_{0,1}, T_{1,0}: \mathcal{F} \rightarrow \mathcal{F}$ by the formulae:

$$
\begin{aligned}
& T_{0,1}(M)=(M \otimes E)_{\gamma-\gamma_{1}} \text { for } M \in \mathcal{F}_{\gamma} \\
& T_{1,0}(M)=(M \otimes E)_{\gamma+\gamma_{1}} \text { for } M \in \mathcal{F}_{\gamma}
\end{aligned}
$$

Next we define isomorphisms of $\mathbb{Z}$-modules $\psi^{ \pm}: \mathcal{K}_{E}(\mathcal{F})^{ \pm} \rightarrow X^{ \pm}$by setting

$$
\psi^{+}(\mathcal{E}(\lambda))=x_{\lambda}, \psi^{-}(\mathcal{E}(\lambda))=0
$$

if $a_{m}=0$,

$$
\psi^{ \pm}\left(\mathcal{E}(\lambda) \pm \mathcal{E}(\lambda)^{\sigma}\right)=x_{\lambda}
$$

if $a_{m}>0$.

Lemma 12. - For any $a>0$ one has

$$
\psi^{ \pm} \circ T_{a+1, a}=F_{a+1, a} \circ \psi^{ \pm}
$$

and

$$
\psi^{ \pm} \circ T_{a, a+1}=F_{a, a+1} \circ \psi^{ \pm} .
$$

If $a=0$, then

$$
\psi^{+} \circ T_{1,0}=F_{1,0} \circ \psi^{+}
$$

and

$$
\psi^{+} \circ T_{0,1}=F_{0,1} \circ \psi^{+} .
$$


Proof. - This lemma can be proven exactly as Lemma 4 by direct comparison.

The action of the functors $T_{a, a+1}$ for $a \geq 1$ in terms of weight diagrams is given in figure 1 .

Below in figure 2 and figure 3 we give the action of $T_{0,1}$ and $T_{1,0}$ respectively.

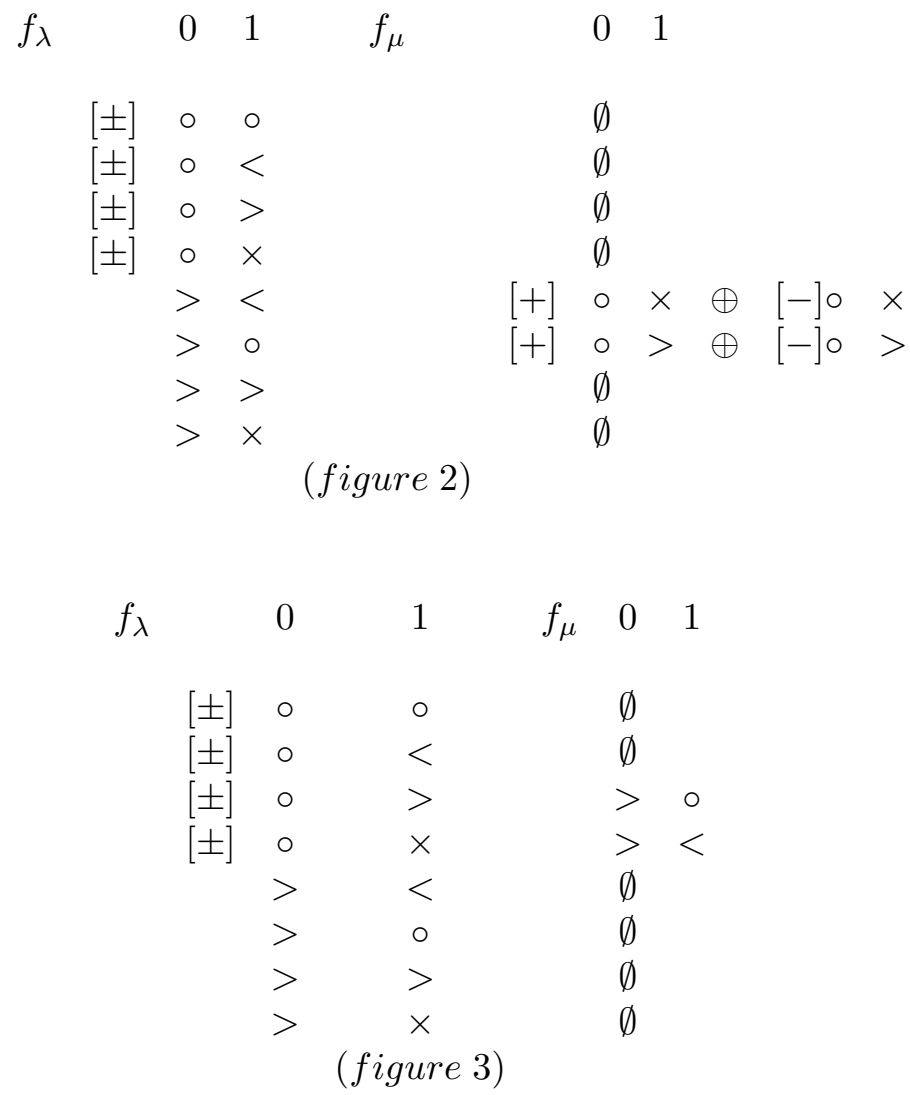

7.2. Action of translation functors on simple modules and PIMs. All statements in this subsection are about all dominant weights, i.e. weights can have tails. The following statement is analogous to Lemma 7.

Lemma 13. - (a) Let $\chi$ and $\theta$ be two distinct central characters such that at $(\chi) \geq$ at $(\theta)$. Assume that $a \geq 1$. Let $T=T_{a, a+1}$ or $T_{a+1, a}$ be a translation functor which maps $\mathcal{F}_{\chi}$ to $\mathcal{F}_{\theta}$. Then, if some dominant weight $\lambda$ (resp. $\lambda_{1}, \lambda_{2}$ ) has central character $\chi$, then $T\left(L_{\lambda}\right)$ is either a simple module of $\mathcal{F}_{\theta}$ or zero.

Moreover, if $T\left(L_{\lambda_{1}}\right)=T\left(L_{\lambda_{2}}\right) \neq 0$, then $\lambda_{1}=\lambda_{2}$.

(b) If at $(\chi) \leq$ at $(\theta)$ and $a \geq 1$, then $T\left(P_{\lambda}\right)$ is either PIM or zero and if $T\left(P_{\lambda_{1}}\right)=$ $T\left(P_{\lambda_{2}}\right) \neq 0$, then $\lambda_{1}=\lambda_{2}$.

We define non-tail elementary changes as a), b), c) in subsection 6.3 with the convention that a non-tail elementary change does not change the sign of the diagram. 
Lemma 14. - Let $\lambda$ be a dominant weight. Assume that the degree of atypicality of $\lambda$ is not less than the degree of atypicality of $\gamma(\lambda)-\gamma_{1}$. If $f_{\lambda}$ does not have $>$ at the tail position, then $T_{0,1}\left(L_{\lambda}\right)=0$. If $f_{\lambda}$ has $>$ at the tail position, then the action of $T_{0,1}$ is given by the following change in the diagram $f_{\lambda}$.

1) ${ }_{x^{k}} \circ \ldots \rightarrow x^{k}>\ldots$;

2) $x_{x^{k-1}} \times \ldots \rightarrow x^{k}>\ldots$;

3) $>\circ \ldots \rightarrow[+] \circ>\ldots \oplus[-] \circ>\ldots$

Assume that the degree of atypicality of $\lambda$ is not less than the degree of atypicality of $\gamma(\lambda)+\gamma_{1}$. If $T_{1,0}\left(L_{\lambda}\right) \neq 0$, then $f_{\lambda}$ has no $>$ at the tail position and $f_{\lambda}(1)=\circ$ or $>$. The action of $T_{1,0}$ is given by the following change in $f_{\lambda}$.

4) $x^{k} \circ \ldots \rightarrow x_{x^{k-1}}^{>}<\ldots$;

5) $x^{k}>\ldots \rightarrow x_{x^{k}}^{>} \circ \ldots$

6) $[ \pm] \circ>\ldots \rightarrow>0 \ldots$

Proof. - The statement is an immediate consequence of Theorem 3.2 (iii) in [25].

Corollary 2. - Let $\lambda$ be dominant and with degree of atypicality not greater than the degree of atypicality of $\gamma(\lambda)+\gamma_{1}$. Then $T_{1,0}\left(P_{\lambda}\right)=0$ if $f_{\lambda}$ contains $>$ at the tail position. If $f_{\lambda}$ does not have $>$ at the tail position the action of $T_{1,0}$ can be described by the following diagrams.

$\left.a^{\prime}\right) x^{k}>\ldots \rightarrow x_{x^{k}}^{>} \ldots \oplus \oplus_{x^{k-1}}^{>} \times \ldots$

$\left.b^{\prime}\right)[ \pm] \circ>\ldots \rightarrow>0 \ldots$

Now assume that the degree of atypicality of $\lambda$ is not greater than the degree of atypicality of $\gamma(\lambda)-\gamma_{1}$. If $T_{0,1}\left(P_{\lambda}\right) \neq 0$ then $f_{\lambda}$ contains $>$ at the tail position. The action of $T_{0,1}$ on $P_{\lambda}$ is given by one of the following changes:

$\left.c^{\prime}\right) \times_{x^{k-1}}^{>}<\ldots \rightarrow x^{k} \circ \ldots$;

$\left.d^{\prime}\right) \underset{x^{k}}{>} \circ \ldots \rightarrow x^{k}>\ldots$;

$\left.e^{\prime}\right)>\circ \ldots \rightarrow[+] \circ>\ldots \oplus[-] \circ>\ldots$

We call a')-e') the elementary tail changes. Note that a translation functor corresponding to an elementary tail change does not always map a PIM to a PIM, a') and e') map sometimes a PIM to the direct sum of two PIMs. Thus, a straightforward analogue of Lemma 11 does not hold.

Lemma 15. - The Grothendieck group $\mathcal{K}_{P}(\mathcal{F})$ is generated (as a $\mathbb{Z}$-module) by $\left[P_{\lambda}\right]$ for typical $\lambda$ and $\left[T_{m} \circ \cdots \circ T_{1}\left(P_{\lambda}\right)\right]$ ( $\lambda$ typical) where $T_{i}$ does not decrease the degree of atypicality of $T_{i-1} \circ \cdots \circ T_{1}\left(P_{\lambda}\right)$.

Proof. - We prove the statement by induction on the degree of atypicality. Let $S$ denote the span of the $\left[T_{m} \circ \cdots \circ T_{1}\left(P_{\lambda}\right)\right]$. It is clear that $\left[P_{\lambda}\right] \in S$ for typical $\lambda$. Assume that $\left[P_{\mu}\right] \in S$ if $a t(\mu)=k-1$. Suppose that $a t(\lambda)=k$. If $f_{\lambda}$ has at least one $\times$ which is not at the tail position, then we can obtain $\left[P_{\lambda}\right]$ from some $\left[P_{\nu}\right]$ with at $(\nu)=k-1$ in the same way as in the proof of Lemma 11.

Now assume that all the $\times$ of $f_{\lambda}$ are at the tail position. We check all the possible cases for $f_{\lambda}$.

If $f_{\lambda}=\times^{k} \circ \ldots$, then $P_{\lambda}=T_{0,1}\left(P_{\nu}\right)$ with $f_{\nu}={ }_{x^{k-1}}^{>}<\ldots$ (Corollary $\left.2 \mathrm{c}^{\prime}\right)$ ). By induction assumption $\left[P_{\nu}\right] \in S$. Hence $\left[P_{\lambda}\right] \in S$.

If $f_{\lambda}=x^{k}>\ldots$ or $f_{\lambda}=x^{k}<\ldots$, we use non-tail elementary changes to move the non-tail symbols to the right, then apply the translation functor as in the previous case 
and then move the non-tail symbols back. For instance, if $f_{\lambda}=x^{k}>0$, we use

$$
\mathrm{x}^{k-1}<>\rightarrow x^{k} \circ>\rightarrow x^{k}>\circ \text {. }
$$

Now let $f_{\lambda}=>_{x^{k}} \ldots$. Using non-tail elementary changes we can reduce to the case $f_{\lambda}={ }_{x^{k}} \circ \ldots$ in the same way as above. By Corollary $\left.2 \mathrm{a}^{\prime}\right)$

$$
T_{1,0}\left(P_{\nu}\right)=P_{\lambda} \oplus P_{\mu},
$$

where $f_{\nu}=\times^{k}>\ldots$ and $f_{\mu}=>_{x^{k-1}}^{>} \times \ldots$ But $f_{\mu}$ has $\times$ at non-tail position. We have proved above that $\left[P_{\mu}\right] \in S$. We also have checked above that $\left[P_{\nu}\right] \in S$. This implies $\left[P_{\lambda}\right] \in S$.

\section{PIM AS A LINEAR COMBINATION OF EulER CHARACTERISTICS}

In this section we compute $a(\lambda, \mu)$ for the orthosymplectic supergroups.

8.1. The case of a tailless weight. In this subsection $G=\operatorname{SOSP}(2 m, 2 n)$ or $\operatorname{SOSP}(2 m+$ $1,2 n)$. We claim that if $\lambda$ is tailless, then $a(\lambda, \mu)$ can be computed in the same way as in the case $G=G L(m, n)$.

Theorem 2. - Let $\lambda$ be a tailless dominant weight. One has:

$$
[P(\lambda)]=\sum_{\mu \in \mathcal{P}(\lambda)} \mathcal{E}(\mu)
$$

Proof. - This statement has the same proof as the corresponding result in the case $\mathfrak{g l}(m, n)$ obtained by J. Brundan. We write down the argument for the sake of completeness.

Due to Lemma 10 and Lemma 15 it is sufficient to check that if the statement holds for $P_{\kappa}$, then it holds for $P_{\lambda}=T\left(P_{\kappa}\right)$, where $T$ is a translation functor corresponding to some elementary change. If the elementary change is of type b) or c) consisting in moving a core symbol from position $t+1$ to $t$, then clearly the weight diagrams of $\mathcal{P}(\lambda)$ are obtained from those of $\mathcal{P}(\kappa)$ by exchanging symbols in position in $t+1$ and $t$. If the elementary change is of type a)

$$
><\rightarrow \times \circ,
$$

then the cap diagram of $f_{\lambda}$ has exactly one new cap joining $\times$ and $\circ$ in $f_{\lambda}$. All other caps remain the same. Hence the statement holds in this case as well.

Remark. As follows from Theorem 2, if $\mu$ is tailless $a(\mu, \nu)=0$ or 1 .

Examples. Let $G=S O S P(7,6)$.

$$
\begin{aligned}
{[P(\circ \times><\times)] } & =\mathcal{E}(\circ \times><\times)+\mathcal{E}(\circ \times><\circ \times)+ \\
& +\mathcal{E}(\circ \circ><\times \circ \times)+\mathcal{E}(\circ \circ><\circ \times \times), \\
{[P(\circ \times \times \circ \times)]=} & \mathcal{E}(\circ \times \times \circ \times)+\mathcal{E}(\circ \times \circ \times \times)+\mathcal{E}(\circ \times \times \circ \circ \circ \times)+ \\
& +\mathcal{E}(\circ \times \circ \times \circ \times)+\mathcal{E}(\circ \circ \times \circ \times \circ \times)+\mathcal{E}(\circ \circ \circ \circ \times \times \circ \times)+ \\
& +\mathcal{E}(\circ \circ \times \circ \circ \times \times)+\mathcal{E}(\circ \circ \circ \times \circ \times \times \times \times) .
\end{aligned}
$$

and the caps are the following: 


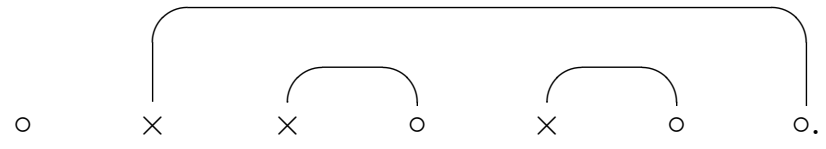

8.2. The general case $G=\operatorname{SOSP}(2 m+1,2 n)$. Here we give an algorithm for calculating $a(\lambda, \mu)$ if $\lambda+\rho \notin \Lambda^{+}$, or, equivalently, if $f_{\lambda}$ has a tail. Color all $\times$ at the tail position. We define the tailless weight $\bar{\lambda}$ as follows. Ignore for a moment the tail position and consider the cap diagram associated to the weight diagram for the remaining positions. We call a position free if it is empty and is not an end of a cap. Enumerate all free position ignoring the others counting from left to right. Move $\times$-s from the tail position to free positions according to the rule below:

- If $f_{\lambda}$ has a core symbol at the tail, move all $\times$ from the tail position to the free positions number $1,3, \ldots$.

- If $f_{\lambda}$ does not have a core symbol at the tail, move all but one $\times$ from the tail to the free positions number $2,4, \ldots$.

Example. If $f_{\lambda}=\left(\times^{3} \times \circ \ldots\right)$, then $f_{\bar{\lambda}}=(\times \times \circ \circ \times \circ \times \circ \ldots)$.

Theorem 3. - Let $G=S O S P(2 m+1,2 n)$. If the tail position of the diagram $f_{\lambda}$ contains $<,>$ or the diagram has $(-)$ sign, then

$$
a(\lambda, \nu)=(-1)^{c(\lambda, \nu)} a(\bar{\lambda}, \nu)
$$

where $c(\lambda, \nu)=x+y$ where $x$ is the total number of colored $\times$ in $f_{\bar{\lambda}}$, and $y$ is the number of colored $\times$ in $f_{\bar{\lambda}}$ moved to the right ends of caps to get $f_{\nu}$ from $f_{\bar{\lambda}}$.

If the sign of $f_{\lambda}$ is $(+)$, we change the sign of $a(\lambda, \nu)$ for all $\nu$ such that $f_{\nu}$ has a $\times$ at the tail position.

Proof. - As in the proof of Theorem 2 we have to check that the statement for $P_{\mu}$ implies the statement for $T\left(P_{\mu}\right)$ for a translation functor $T$ corresponding to some elementary change. This check for elementary changes a)-c) is completely analogous to the case of tailless $\lambda$. So we leave it to the reader.

Now we deal with the tail elementary changes at)-ct). Let $\lambda$ and $\mu$ be related by an elementary change bt) i.e.

$$
f_{\mu}=(-) \times^{k}>\ldots \rightarrow f_{\lambda}=x^{k} \circ \ldots
$$

Then $\bar{\lambda}$ is obtained from $\bar{\mu}$ by switching $\times$ at the tail position with $>$ at position $\frac{3}{2}$, and the number of colored $\times$ in $f_{\bar{\lambda}}$ and in $f_{\bar{\mu}}$ is the same. All $\nu^{\prime} \in \mathcal{P}(\bar{\lambda})$ are obtained from $\nu \in \mathcal{P}(\bar{\mu})$ by interchanging symbols at positions $\frac{1}{2}$ and $\frac{3}{2}$. Clearly $c(\lambda, \nu)=c(\mu, \nu)$. The case of elementary change ct) is similar.

Now let $\lambda$ and $\mu$ be related by an elementary change at), namely

$$
f_{\mu}=>_{x^{k}}^{>}<\ldots \rightarrow f_{\lambda}=(+) x^{k+1} \circ \ldots
$$

Then $\bar{\lambda}$ is obtained from $\bar{\mu}$ by removing core symbols from $\frac{1}{2}$ and $\frac{3}{2}$ and adding $\times$ to the tail position $\frac{1}{2}$. The cap diagram for $f_{\bar{\lambda}}$ has an additional cap joining $\frac{1}{2}$ and $\frac{3}{2}$. The number of colored $\times$ increases by 1 . However, since sign of $f_{\lambda}$ is $(+)$, the signs agree after applying the switch functor to $P_{\lambda}$. 
Examples. $G=\operatorname{SOSP}(5,4)$

$$
\begin{aligned}
& {\left[P\left(\begin{array}{cc}
> & \\
\times & <
\end{array}\right)\right]=-\mathcal{E}(><\times)+\mathcal{E}(><\circ \times),} \\
& {\left[P\left(\begin{array}{cc}
(+) & \times \\
& \times
\end{array}\right)\right]=-\mathcal{E}((+) \times \circ \times)-\mathcal{E}(\circ \times \times)+\mathcal{E}((+) \times \circ \circ \times)+\mathcal{E}(\circ \times \circ \times),} \\
& {\left[P\left(\begin{array}{cc}
(-) & \times \\
& \times
\end{array}\right)\right]=\mathcal{E}((+) \times \circ \times)-\mathcal{E}(\circ \times \times)-\mathcal{E}((+) \times \circ \circ \times)+\mathcal{E}(\circ \times \circ \times) .}
\end{aligned}
$$

8.3. The general case $G=S O S P(2 m, 2 n)$. It turns out that the coefficients $a(\lambda, \mu)$ for $\operatorname{SOSP}(2 m, 2 n)$ can be expressed in terms of certain $a\left(\lambda^{\prime}, \mu^{\prime}\right)$ for $\operatorname{SOSP}(2 m+1,2 n)$. Let $\lambda$ be a dominant weight for $\operatorname{SOSP}(2 m, 2 n)$. Let $\lambda^{\prime}$ be the weight for $\operatorname{SOSP}(2 m+1,2 n)$ such that the corresponding weight diagrams are related by

$$
f_{\lambda^{\prime}}(t)=f_{\lambda}\left(t-\frac{1}{2}\right)
$$

If $f_{\lambda}$ has some $\times$ at the tail and no $>$, then we assume that $f_{\lambda^{\prime}}$ has $(+)$ sign.

Note that if $\nu+\rho \in \Lambda^{+}$, then $\nu^{\prime}$ has either $>$ or $\circ$ at the tail position. Note also that the map $\lambda \rightarrow \lambda^{\prime}$ is neither injective nor surjective, since $(\sigma(\lambda))^{\prime}=\lambda^{\prime}$ and $f_{\nu^{\prime}}$ does not have $<$ at the tail position. If $\nu$ is tailless, then $\nu^{\prime}$ is also tailless, however some weights with a tail are mapped to the tailless weights. For instance $(\times \times \circ \ldots)$ has a tail for $\operatorname{SOSP}(2 m, 2 n)$ but the corresponding weight diagram $(+)(\times \times \circ \ldots)$ is the diagram of a tailless weight for $\operatorname{SOSP}(2 m+1,2 n)$.

Theorem 4. - Let $G=\operatorname{SOSP}(2 m, 2 n)$. Then for any dominant $\nu$ and $\lambda$ such that $\nu+\rho \in \Lambda^{+}, \lambda+\rho \notin \Lambda^{+}$, we have

$$
a(\lambda, \nu)=a\left(\lambda^{\prime}, \nu^{\prime}\right)
$$

Remark. - The case of a tailless $\lambda$ was covered in Theorem 2. Theorem 4 implies the following relation in the Grothendieck group of $\operatorname{SOSP}(2 m, 2 n)$ :

$$
\left[P_{\lambda}\right]=\sum_{\nu+\rho \in \Lambda^{+}} a\left(\lambda^{\prime}, \nu^{\prime}\right) \mathcal{E}(\nu)
$$

The right hand side of this expression is $\sigma$-invariant which is consistent with the condition $P_{\lambda}^{\sigma}=P_{\lambda}$.

Proof. - Let us introduce a few notations. For any $\mathbb{Z}$-module $A$, denote $\mathbb{Q} A=\mathbb{Q} \otimes_{\mathbb{Z}} A$. For $m, n$ fixed, we denote by $\mathcal{K}^{+}(\mathcal{F})$ the $\sigma$-invariant subgroup of the Grothendieck group for $\operatorname{SOSP}(2 m, 2 n)$ and by $\mathcal{K}\left(\mathcal{F}^{\prime}\right)$ the Grothendieck group for $\operatorname{SOSP}(2 m+1,2 n)$. The definitions of $\mathcal{K}_{P}^{+}(\mathcal{F}), \mathcal{K}_{E}^{+}(\mathcal{F}), \mathcal{K}_{P}\left(\mathcal{F}^{\prime}\right)$ and $\mathcal{K}_{E}\left(\mathcal{F}^{\prime}\right)$ are obvious.

We define two $\mathbb{Q}$-linear maps $\alpha: \mathbb{Q}_{E}\left(\mathcal{F}^{\prime}\right) \rightarrow \mathbb{Q}_{E} \mathcal{K}_{E}^{+}(\mathcal{F})$ and $\beta: \mathbb{Q}_{E}(\mathcal{F}) \rightarrow \mathbb{Q}_{E} \mathcal{K}_{E}\left(\mathcal{F}^{\prime}\right)$ in the following way. First we define $\alpha: U \rightarrow V \oplus V^{*}$ and $\beta: V \oplus V^{*} \rightarrow U$ by the formulae

$$
\begin{gathered}
\alpha\left(v_{1 / 2}\right)=0, \alpha\left(v_{i}\right)=u_{i-1 / 2}, i>1, \alpha\left(w_{i}\right)=u_{1 / 2-i} \\
\beta\left(u_{i}\right)=w_{1 / 2-i}, i \leq 0, \beta\left(u_{i}\right)=v_{i-1 / 2}, i>0 .
\end{gathered}
$$


Next, we extend $\alpha$ and $\beta$ to $\Lambda^{m}\left(V^{*}\right) \otimes \Lambda^{n}(V)$ and $X^{+}$in the natural way. The following diagram explains the maps $\alpha$ and $\beta$ on the level of Grothendieck groups

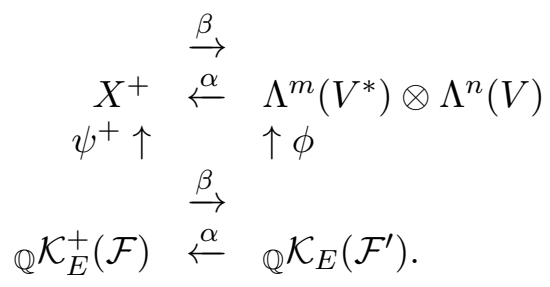

Note that $\alpha$ is surjective, $\beta$ is injective and $\alpha \circ \beta=$ id. For a linear operator $T$ in $\mathcal{K}_{E}^{+}(\mathcal{F})$ corresponding to a translation functor we define an operator $T^{\prime}$ in $\mathcal{K}_{E}\left(\mathcal{F}^{\prime}\right)$ by the following rules

if $T=T_{a, a+1}$ then $T^{\prime}=T_{a+1 / 2, a+3 / 2}$,

if $T=T_{a, a-1}$ and $a \neq 1$ then $T^{\prime}=T_{a+1 / 2, a-1 / 2}$,

if $T=T_{1,0}$ then $T^{\prime}=2 T_{3 / 2,1 / 2}$.

Direct computation gives the following result:

Lemma 16. - One has for any $T=T_{a, a \pm 1}$

$$
T=\alpha \circ T^{\prime} \circ \beta .
$$

Next we define $\bar{\beta}: \mathbb{Q}_{P}^{+}(\mathcal{F}) \rightarrow \mathbb{Q}_{P}\left(\mathcal{F}^{\prime}\right)$ by setting:

$\bar{\beta}\left[P_{\lambda}\right]=\frac{1}{2}\left(\left[P_{\lambda^{\prime}}\right] \oplus\left[s w\left(P_{\lambda^{\prime}}\right)\right]\right)$ if $f_{\lambda}\left(\frac{1}{2}\right)=\times^{k}, k>0$ (we use the notation $\times^{k}$ for the multiset containing $\times k$ times);

$\bar{\beta}\left[P_{\lambda} \oplus P_{\lambda}^{\sigma}\right]=\left[P_{\lambda^{\prime}}\right]$ if $f_{\lambda}\left(\frac{1}{2}\right)=\circ ;$

$\bar{\beta}\left[P_{\lambda}\right]=\left[P_{\lambda^{\prime}}\right]$ if $f_{\lambda}\left(\frac{1}{2}\right)=\left\{x^{k},>\right\}, k \geq 0 ;$

and $\bar{\alpha}: \mathbb{Q}_{P}\left(\mathcal{F}^{\prime}\right) \rightarrow \mathbb{Q}_{P}^{+}(\mathcal{F})$ as follows:

If $\nu=\lambda^{\prime}$ or $s w\left(\lambda^{\prime}\right)$ for some dominant $\operatorname{SOSP}(2 m, 2 n)$ weight $\lambda$ we set $\bar{\alpha}\left[P_{\nu}\right]=\left[P_{\lambda}\right]$ or $\left[P_{\lambda} \oplus P_{\lambda}^{\sigma}\right]$ (in the case $P_{\lambda} \neq P_{\lambda}^{\sigma}$ ). Otherwise we set $\bar{\alpha}\left[P_{\nu}\right]=0$.

It follows immediately from the definitions that $\bar{\alpha}$ is surjective, $\bar{\beta}$ is injective and $\bar{\alpha} \circ \bar{\beta}=$ id.

Lemma 17. - Let $T=T_{a, a \pm 1}, P=P_{\lambda}$ or $P=P_{\lambda} \oplus P_{\lambda}^{\sigma}$. If $T$ does not decrease the degree of atypicality of $\lambda$, then

$$
T([P])=\bar{\alpha} \circ T^{\prime} \circ \bar{\beta}([P]) .
$$

Proof. - If $T \neq T_{0,1}$ or $T_{1,0}$ the statement follows from the fact that the elementary changes listed in subsection 6.3 are the same in the even and odd cases.

If $T \neq T_{0,1}$ or $T_{1,0}$ one can just compare the actions of $T$ and $T^{\prime}$ in all possible cases. We show how it works in the most interesting cases and leave to the reader the remaining cases.

$$
\begin{aligned}
& x^{k}<\stackrel{\bar{\beta}}{\rightarrow} \frac{1}{2}\left((+) \times^{k}<\oplus(-) \times^{k}<\right) \\
& T_{0,1} \downarrow \quad \downarrow T_{1 / 2,3 / 2} \\
& 0 \stackrel{\bar{\alpha}}{\leftarrow} \quad \frac{1}{2}\left(\begin{array}{l}
<k \\
x^{k}
\end{array}\right) \circ \\
& >_{x^{k-1}}<\stackrel{\bar{\beta}}{\rightarrow} \underset{x^{k-1}}{>}< \\
& T_{0,1} \downarrow \quad \downarrow T_{1 / 2,3 / 2} \\
& x^{k} \circ \stackrel{\bar{\alpha}}{\leftarrow}(+) x^{k} \circ
\end{aligned}
$$




$$
\begin{aligned}
& \times^{k}>\quad \stackrel{\bar{\beta}}{\rightarrow} \quad \frac{1}{2}\left((+) x^{k}>\oplus(-) x^{k}>\right) \\
& T_{1,0} \downarrow \quad \downarrow 2 T_{3 / 2,3 / 2} \\
& >_{x^{k}} \circ \oplus_{x^{k-1}}^{>} \times \stackrel{\bar{\alpha}}{\leftarrow} \underset{x^{k}}{>} \circ \bigoplus_{x^{k-1}}^{>} \times \\
& {[+] \circ>\oplus[-] \circ>\stackrel{\bar{\beta}}{\rightarrow} \circ>} \\
& \downarrow T_{1,0} \quad \downarrow 2 T_{3 / 2,1 / 2} \\
& 2>0 \quad \stackrel{\bar{\alpha}}{\leftarrow} 2>0
\end{aligned}
$$

Lemma 18. - One has

$$
\left.\beta \circ \alpha\right|_{\mathbb{Q} \mathcal{K}_{P}\left(\mathcal{F}^{\prime}\right)}=\bar{\beta} \circ \bar{\alpha}
$$

Proof. - Both $\beta \circ \alpha$ and $\bar{\beta} \circ \bar{\alpha}$ are projectors. Observe that $\operatorname{Ker} \beta \circ \alpha$ is generated by $\left\{\mathcal{E}(\lambda) \mid f_{\lambda}\left(\frac{1}{2}\right)=<\right.$ or $\left.\times\right\}$. Ker $\bar{\beta} \circ \bar{\alpha}$ is generated by $\left\{\left[P_{\lambda}\right] \mid f_{\lambda}\left(\frac{1}{2}\right)=\left\{x^{k},<\right\}\right\}$ and $\left\{\left[P_{\lambda}\right]-\right.$ $\left.\left[\operatorname{sw}\left(P_{\lambda}\right)\right] \mid f_{\lambda}\left(\frac{1}{2}\right)=\times^{k}\right\}$. Now we can use Theorem 3. If $f_{\lambda}\left(\frac{1}{2}\right)$ contains $<$, then $\left[P_{\lambda}\right]=$ $\sum a(\lambda, \mu) \mathcal{E}(\mu)$ with $f_{\mu}\left(\frac{1}{2}\right)=<$, and $\alpha(\mathcal{E}(\mu))=0$. If $f_{\lambda}\left(\frac{1}{2}\right)=x^{k}$, then $\left[P_{\lambda}\right]-\left[\operatorname{sw}\left(P_{\lambda}\right)\right]=$ $\sum a(\lambda, \mu) \mathcal{E}(\mu)$ with $f_{\mu}\left(\frac{1}{2}\right)=\times$. Again $\alpha(\mathcal{E}(\mu))=0$. That implies

$$
\operatorname{Ker} \bar{\beta} \circ \bar{\alpha}=\operatorname{Ker} \beta \circ \alpha \cap \mathbb{Q}_{\mathcal{P}}\left(\mathcal{F}^{\prime}\right) \text {. }
$$

Similarly

$$
\operatorname{Im} \bar{\beta} \circ \bar{\alpha}=\operatorname{Im} \beta \circ \alpha \cap{ }_{\mathbb{Q}} \mathcal{K}_{P}\left(\mathcal{F}^{\prime}\right)
$$

The statement follows.

Lemma 19. - One has

$$
\left.\beta\right|_{\mathbb{Q} \mathcal{K}_{P}^{+}(\mathcal{F})}=\bar{\beta},\left.\alpha\right|_{\mathbb{Q}} \mathcal{K}_{P}\left(\mathcal{F}^{\prime}\right)=\bar{\alpha}
$$

Proof. - By definition $\beta([P])=\bar{\beta}([P])$ if $P$ is typical or a direct sum of two typical PIMs. By Lemma 15 it is sufficient to check that $\beta([P])=\bar{\beta}([P])$ implies $\beta(T[P])=\bar{\beta}(T[P])$ if $T$ does not decrease the degree of atypicality of $P$. Indeed we have

$$
\bar{\beta}(T([P]))=\bar{\beta} \circ \bar{\alpha}\left(T^{\prime}(\bar{\beta}([P]))\right)=\bar{\beta} \circ \bar{\alpha}\left(T^{\prime}(\beta([P]))\right)
$$

and similarly

$$
\beta(T([P]))=\beta \circ \alpha\left(T^{\prime}(\beta([P]))\right) .
$$

Thus the statement about $\beta$ follows from Lemma 17 .

The statement about $\alpha$ follows immediately from Lemma 18 and the statement about $\beta$. Indeed, for any $P \in \mathcal{K}_{P}\left(\mathcal{F}^{\prime}\right)$ we have

$$
\alpha(P)=\alpha \circ \beta \circ \alpha(P)=\alpha \circ \bar{\beta} \circ \bar{\alpha}(P)=\alpha \circ \beta \circ \bar{\alpha}(P)=\bar{\alpha}(P) .
$$

Now we are ready to prove Theorem 4 . Write

$$
\left[P_{\lambda}\right]=\alpha\left(\beta\left(\left[P_{\lambda}\right]\right) .\right.
$$

Use Theorem 3. If $f_{\lambda}$ has $>$ at the tail we have

$$
\beta\left(\left[P_{\lambda}\right]\right)=\left[P_{\lambda^{\prime}}\right]=\sum a\left(\lambda^{\prime}, \nu^{\prime}\right) \mathcal{E}\left(\nu^{\prime}\right),
$$


and since $\alpha\left(\mathcal{E}\left(\nu^{\prime}\right)\right)=\mathcal{E}(\nu)$ we obtain

$$
\left[P_{\lambda}\right]=\sum a\left(\lambda^{\prime}, \nu^{\prime}\right) \mathcal{E}(\nu) .
$$

If $f_{\lambda}$ does not have $>$ at the tail position, then

$$
\beta\left(\left[P_{\lambda}\right]\right)=\frac{1}{2}\left[P_{\lambda^{\prime}} \oplus s w\left(P_{\lambda^{\prime}}\right)\right]=\sum a\left(\lambda^{\prime}, \nu^{\prime}\right) \mathcal{E}\left(\nu^{\prime}\right) .
$$

Here all $f_{\nu^{\prime}}$ have $\circ$ at the tail position. Hence again

$$
\left[P_{\lambda}\right]=\sum a\left(\lambda^{\prime}, \nu^{\prime}\right) \mathcal{E}(\nu) .
$$

Examples. $G=S O S P(4,2)$.

$$
\begin{aligned}
& {[P(>><)]=\mathcal{E}(>><),} \\
& {[P(>\times)]=\mathcal{E}(>\times \circ)+\mathcal{E}(>\circ \times),} \\
& {[P(><>)]=\mathcal{E}(><>),} \\
& {[P(\times \circ>)]=\mathcal{E}([+] \circ \times>)+\mathcal{E}([-] \circ \times>),} \\
& {[P(\times>\circ)]=\mathcal{E}([+] \circ>\times)+\mathcal{E}([-] \circ>\times),} \\
& {[P(>\circ \circ)+P(>\times \circ)]=2 \mathcal{E}(>\circ \times),} \\
& {[P(\times \circ \circ)]=-\mathcal{E}(>\times \circ)+\mathcal{E}(>\circ \times) .} \\
& G=S O S P(4,4) \\
& {\left[P\left(\begin{array}{c}
\times \\
\times
\end{array}\right)\right]=-\mathcal{E}([+] \circ \times \times)-\mathcal{E}([-] \circ \times \times)+\mathcal{E}([+] \circ \times \circ \times)+\mathcal{E}([-] \circ \times \circ \times) .}
\end{aligned}
$$

Corollary 3. - Let $G=S O S P(2 m, 2 n)$ or $S O S P(2 m+1,2 n)$. Then for any dominant $\lambda, \mu$ such that $\mu+\rho \in \Lambda^{+}$we have $a(\lambda, \mu)=0$ or \pm 1 . If $\lambda+\rho \in \Lambda^{+}$, then $a(\lambda, \mu) \geq 0$.

8.4. A basis in $\left(\Lambda^{m}\left(V^{*}\right) \otimes \Lambda^{n}(V)\right)_{\mathbb{Q}}$. We assume here that $G=S O S P(2 m+1,2 n)$. The goal of this subsection is to show that $\varphi\left[P_{\lambda}\right]$ for all dominant $\lambda$ form a basis in $\Lambda^{m \mid n}:=\left(\Lambda^{m}\left(V^{*}\right) \otimes \Lambda^{n}(V)\right)_{\mathbb{Q}}$. It follows from [23] that $\Lambda^{m \mid n}$ is an indecomposable $\mathfrak{g l}_{\infty / 2^{-}}$ module. Let $\Theta: \Lambda^{m \mid n} \rightarrow \Lambda^{m-1 \mid n-1}$ be the contraction map defined by

$$
\begin{gathered}
\Theta\left(w_{a_{1}} \wedge \cdots \wedge w_{a_{m}} \otimes v_{b_{1}} \wedge \cdots \wedge v_{b_{n}}\right)= \\
\sum_{i \leq m, j \leq n}(-1)^{i+j}\left\langle w_{a_{i}}, v_{b_{j}}\right\rangle w_{a_{1}} \wedge \cdots \wedge w_{a_{i-1}} \wedge w_{a_{i+1}} \wedge \cdots \wedge w_{a_{m}} \otimes v_{b_{1}} \wedge \cdots \wedge v_{b_{j-1}} \wedge v_{b_{j+1}} \wedge \cdots \wedge v_{b_{n}} .
\end{gathered}
$$

Then

$$
0 \subset \operatorname{Ker} \Theta \subset \operatorname{Ker} \Theta^{2} \cdots \subset \operatorname{Ker} \Theta^{\min (m, n)} \subset \Lambda^{m \mid n}
$$

defines the socle filtration on $\Lambda^{m \mid n}$.

Let $\lambda$ be a tailless weight and $\Theta^{\lambda}$ be the set of all weights $\mu$ such that $f_{\mu}$ is obtained from $f_{\lambda}$ by removing one $\times$. Let $\mu \in \Theta^{\lambda}$. If $f_{\mu}$ is obtained from $f_{\lambda}$ by removing a $\times$ at position $t$, then by $s(\mu)$ we denote the total number of $>,<$ standing before $t$ plus $t+\frac{1}{2}$. By a direct check for any tailless $\lambda$ we have

$$
\Theta\left(x_{\lambda}\right)=\sum_{\mu \in \Theta^{\lambda}}(-1)^{s(\mu)} x_{\mu}
$$

Now let $\lambda$ be any dominant weight, $k$ be the length of the tail of $\lambda$ and $c(\lambda)$ be the tailess weight obtained from $\lambda$ by removing the $k \times$-s from the tail position, getting a dominant weight for $\operatorname{SOSP}(2 m+1-2 k, 2 n-2 k)$. By $P_{c(\lambda)}$ we denote the indecomposable 
projective module over $\operatorname{SOSP}(2 m+1-2 k, 2 n-2 k)$ with highest weight $c(\lambda)$. It is not hard to show from the above formula and Theorem 3 that

$$
\Theta^{k+1}\left(\varphi\left[P_{\lambda}\right]\right)=0, \Theta^{k}\left(\varphi\left[P_{\lambda}\right]\right)=2^{k} k ! \varphi\left[P_{c(\lambda)}\right] .
$$

Proposition 3. The set $\varphi\left[P_{\lambda}\right]$ for all dominant $\lambda$ forms a basis of $\Lambda^{m \mid n}$ over $\mathbb{Q}$. Moreover, $\varphi\left[P_{\lambda}\right]$ for all dominant $\lambda$ with tail length less or equal than $k$ form a basis of the $(1+k)$-th term $\operatorname{Ker} \Theta^{k+1}$ of the socle filtration.

Proof. It is sufficient to show that the set $\varphi\left[P_{\lambda}\right]$ for all tailless $\lambda$ is a basis of $\operatorname{Ker} \Theta$, since then the statement follows from (8) and (9) by induction.

The linear independence of $\varphi\left[P_{\lambda}\right]$ for all tailless $\lambda$ follows from the fact that $\varphi\left[P_{\lambda}\right]=$ $x_{\lambda}+\sum x_{\mu}$ for some $\mu>\lambda$.

The fact that $\varphi\left[P_{\lambda}\right]$ for all tailless $\lambda$ generate $\operatorname{Ker} \Theta$ follows from irreducibility of $\operatorname{Ker} \Theta$ over $\mathfrak{g l}_{\infty / 2}$. Indeed, a vector $x_{\nu}$ for some typical $\nu$ generate $\operatorname{Ker} \Theta$ over $U\left(\mathfrak{g l}_{\infty / 2}\right)$. Hence any vector in $\operatorname{Ker} \Theta$ is a finite linear combination $\sum a_{j} Y_{j} x_{\nu}$ where $a_{j} \in \mathbb{Q}$ and $Y_{j}$ are non-commutative monomials in $E_{a, a \pm 1}$. Since $x_{\nu}=\varphi\left[P_{\nu}\right]$ and translation functors map projective modules to projective modules, we have that $Y_{j} x_{\nu}$ is a finite positive linear combination of $\varphi\left[P_{\lambda}\right]$-s. The statement follows.

Corollary 4. In the abelian group $\mathcal{K}_{E}(\mathcal{F}) / \mathcal{K}_{P}(\mathcal{F})$, every element has finite order.

Now let us illustrate the above statement on the example of $\operatorname{SOSP}(3,2)$. Tailess dominant highest weight are of the form $a \varepsilon+b \delta$ with $a-1, b \in \mathbb{Z}_{\geq 0}$. The only dominant weight with a tail is 0 . We can express any Euler characteristic class $\mathcal{E}(\mu)$ as a finite rational linear combination of projective modules. If $a-1 \neq b$ we have $\mathcal{E}(a, b)=\left[P_{a, b}\right]$ and if $a-1=b$ we obtain

$$
\begin{gathered}
\mathcal{E}(1,0)=\frac{1}{2}\left(\left[P_{1,0}\right]-\left[P_{0}\right]\right), \mathcal{E}(2,1)=\frac{1}{2}\left(\left[P_{1,0}\right]+\left[P_{0}\right]\right), \\
\mathcal{E}(a+1, a)=\sum_{j=1}^{a-1}(-1)^{j+a-1}\left[P_{j+1, j}\right]-\frac{(-1)^{a}}{2}\left(\left[P_{1,0}\right]+\left[P_{0}\right]\right) .
\end{gathered}
$$

To write the classes of simple modules $\left[L_{\lambda}\right]$ in terms of $\mathcal{E}(\mu)$ ( $\lambda$ is dominant, $\mu$ is tailless) one can use BGG resiprocity. However, it is not possible to construct a basis dual to $\left[P_{\lambda}\right]$ in $\Lambda^{m \mid n}$. One can construct such a basis in a natural completion $\bar{\Lambda}^{m \mid n}:=\left(\Lambda^{n \mid m}\right)^{\prime} \supset \Lambda^{m \mid n}$, where by $\left(\Lambda^{n \mid m}\right)^{\prime}$ we mean the algebraic dual of $\Lambda^{n \mid m}$. For our example we have

$$
\begin{aligned}
& {\left[L_{0}\right]=\frac{1}{2}\left(-\mathcal{E}(1,0)-\sum_{j=1}^{\infty}(-1)^{j} \mathcal{E}(j+1, j)\right),} \\
& {\left[L_{1,0}\right]=\frac{1}{2}\left(\mathcal{E}(1,0)-\sum_{j=1}^{\infty}(-1)^{j} \mathcal{E}(j+1, j)\right),}
\end{aligned}
$$

and for $a>0$

$$
\left[L_{a+1, a}\right]=\sum_{j=a+1}^{\infty}(-1)^{a+j+1} \mathcal{E}(j+1, j)
$$




\section{REFERENCES}

[1] P. N. Achar, C. Stroppel, Completions of Grothendieck groups. arXiv:1105.2715.v.2.

[2] J. Bernstein, I. Gel'fand, S. Gel'fand, Category of $\mathfrak{g}$-modules. Func. Anal. Appl., 10 (1976), 87-92.

[3] R. Bott, Homogeneous vector bundles. Annals of Mathematics, 66 (1957), no. 2.

[4] J. Brundan, Kazhdan-Lusztig polynomials and character formulae for the Lie superalgebra $g l(m \mid n)$. J. Amer. Math. Soc. 16 (2003), no. 1, 185-231.

[5] R. Brauer, On modular and p-adic representations of algebras. Proc. Nat. Acad. Sci. USA, 25. (1939), $252-258$.

[6] J. Brundan, C. Stroppel, Highest weight categories arising from Khovanov's diagram algebra I: cellularity. Preprint, 2008.

[7] J. Brundan, C. Stroppel, Highest weight categories arising from Khovanov's diagram algebra II: Koszulity. Transform. Groups 15 (2010), 1-45.

[8] J. Brundan, C. Stroppel, Highest weight categories arising from Khovanov's diagram algebra III: Category $\mathcal{O}$. To appear in Represent. Theory (2011).

[9] J. Brundan, C. Stroppel, Highest weight categories arising from Khovanov's diagram algebra IV: the general linear supergroup. To appear in J. Eur. Math. Soc. (2011),

[10] S.-J. Chang, N. Lam, W. Wang, Super duality and irreducible characters of ortho-symplectic Lie superalgebras. Inventiones Mathematicae, to appear (2011) (http://www.springerlink.com/content/y4633362461h8365).

[11] E. Cline, B. Parshall, L. Scott, Finite-dimensional algebras and highest weight categories. J. Reine Agnew. Math., 391 (1988), 85-99.

[12] C. Gruson, V. Serganova, Cohomology of generalized supergrassmannians and character formulae for basic classical Lie superalgebras. Proc. of the LMS, 101 (2010), no.3, 852-892.

[13] J. Humphreys, Modular representation of classical Lie algebras and semisimple groups. J. Algebra, 19 (1971), 51-79.

[14] R. Irving, BGG algebra and BGG reciprocity principle. J. Algebra, 135 (1990), 363-380.

[15] J. Jantzen, Representations of algebraic groups. Second edition. Mathematical Surveys and Monographs, 107. American Mathematical Society, Providence, RI, 2003.

[16] V. Kac, Lie superalgebras. Advances in Math. 26 (1977), no. 1, 8-96.

[17] V. Kac, Characters of typical representations of classical Lie superalgebras. Comm. Algebra 5 (1977), no. 8, 889-897.

[18] Yu. Manin, Gauge field theory and complex geometry. Translated from the 1984 Russian original by N. Koblitz and J. R. King. Grundlehren der Mathematischen Wissenschaften [Fundamental Principles of Mathematical Sciences], 289. Springer-Verlag, Berlin, 1997.

[19] Yu. Manin, I. Penkov, A. Voronov, Elements of supergeometry. (Russian) Translated in J. Soviet Math. 51 (1990), no. 1, 2069-2083.

[20] I. Musson, Lie superalgebras and enveloping algebra, American Mathematical Society, Providence, RI, 2012.

[21] I. Musson, V. Serganova, Combinatorics of character formulas for the Lie superalgebra $\mathfrak{g l}(m, n)$. Transformation groups, 16 (2011), no. 2, 555-578.

[22] I. Penkov, Borel-Weil-Bott theory for classical Lie supergroups. (Russian) Translated in J. Soviet Math. 51 (1990), no. 1, 2108-2140.

[23] I. Penkov, K. Styrkas, Tensor representations of infinite-dimensional root-reductive Lie algebras. Developments and trends in infinite-dimensional Lie theorey, Progr. in Math., Birkhauser, 2011, 127-150.

[24] V. Serganova, Kazhdan-Lusztig polynomials and character formula for the Lie superalgebra $g l(m \mid n)$. Selecta Math. (N.S.) 2 (1996), no. 4, 607-651.

[25] V. Serganova, On the superdimension of a finite-dimensional representation of a basic classical Lie superalgebra. Supersymmetry in mathematics and physics. LNM. To appear.

[26] V. Serganova, Quasireductive supergroups. New Developments in Lie theory and its applications, Contemprorary Mathematics, AMS, 544, 2011, 141-159.

[27] V. Serganova, Structure and representation theory of Kac-Moody superalgebras. Highlights in Lie algebraic methods, Birkhauser, 2011, to appear.

[28] V. Serganova, Kac-Moody superalgebras and integrability. Developments and trends in infinitedimensional Lie theorey, Progr. in Math., Birkhauser, 2011.

[29] A. Sergeev, A. Veselov. Grothendieck ring of basic classical Lie superalgebras. Annals of Mathematics, 173 (2011), no.2. 
[30] Y. M. Zou, Categories of finite-dimensional weight modules over type I classical Lie superalgebras. J. of Algebra, 180 (1996),459-482. 